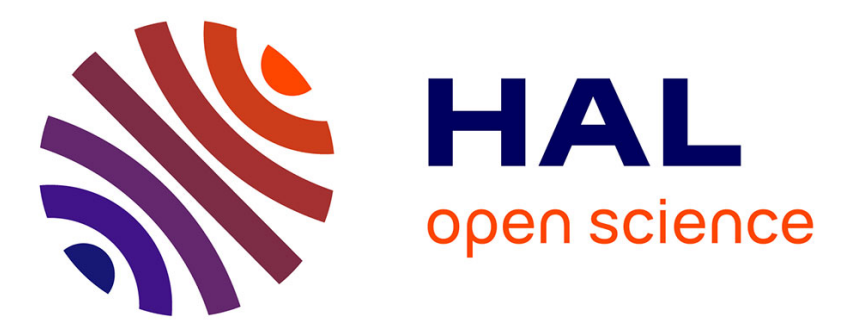

\title{
Thermomechanical analysis of the onset of strain concentration zones in wet polyamide 6.6 subjected to cyclic loading
}

\author{
Adil Benaarbia, André Chrysochoos, Gilles Robert
}

\section{- To cite this version:}

Adil Benaarbia, André Chrysochoos, Gilles Robert. Thermomechanical analysis of the onset of strain concentration zones in wet polyamide 6.6 subjected to cyclic loading. Mechanics of Materials, 2016, 99, pp.9-25. 10.1016/j.mechmat.2016.04.011 . hal-01325033

\section{HAL Id: hal-01325033 \\ https://hal.science/hal-01325033}

Submitted on 2 Jun 2016

HAL is a multi-disciplinary open access archive for the deposit and dissemination of scientific research documents, whether they are published or not. The documents may come from teaching and research institutions in France or abroad, or from public or private research centers.
L'archive ouverte pluridisciplinaire $\mathbf{H A L}$, est destinée au dépôt et à la diffusion de documents scientifiques de niveau recherche, publiés ou non, émanant des établissements d'enseignement et de recherche français ou étrangers, des laboratoires publics ou privés. 


\title{
Thermomechanical analysis of the onset of strain concentration zones in wet polyamide 6.6 subjected to cyclic loading
}

\author{
Adil Benaarbia, André Chrysochoos, Gilles Robert \\ University of Montpellier, Mechanics and Civil Engineering Laboratory, \\ CC 048, Place E. Bataillon, 34095 Montpellier, France
}

Solvay Engineering Plastics, Centre de Simulation et Validation des Applications, Avenue de Ramboz - BP64 - 69192 Saint Fons, France

\begin{abstract}
:
In the present paper, we put forward some experimental results on the local thermomechanical behavior of a wet PA6.6 matrix subjected to cyclic loading. We used photomechanical techniques to assess the creep and cyclic strain rate fields, as well as the associated dissipation and coupling heat source fields. This thermomechanical analysis enabled us, from the very beginning of the cyclic test, to track the onset of strain concentration zones and note their limited development. Moreover, this analysis helped us to highlight the co-existence of glassy, rubber and mixed thermal responses. This revealed that wet PA6.6 was a graded material whose property variations surely promoted the development of strain concentration.
\end{abstract}

Keywords: polyamide cyclic behavior, strain concentration, dissipation fields, thermoelastic inversion.

\section{Introduction}

Polyamides (PAs) belong to the wide variety of semi-crystalline thermoplastics that are being increasingly used in various applications such as automotive, aerospace and many others. These engineering materials have received much attention because of their low density, ease of manufacture and deformability as compared to corresponding standard materials. However, PAs exhibit various deformation mechanisms (e.g. rate dependence and strong coupling effects, ratcheting, internal energy storage and release, damage, etc.), which are highly influenced by hygro-thermomechanical boundary and loading conditions (Benaarbia et al., 2014a,b; Arif et al., 2014). Indeed, the mechanical behavior of this type of material is particularly sensitive to its thermal and moisture states. Some of these mechanisms have already been underlined in previous experimental investigations, such as (Horst and Spoormaker, 1996; Bernasconi and Kulin, 2009; Launay et al., 2011; Launay et al., 2013). Further insight is, however, still required on some issues, especially concerning the local analysis of deformation mechanisms boosted by the dissipative effects and competition between standard and entropic thermoelastic couplings, which in turn are highly influenced by the moisture, temperature and strain distribution.

The cyclic behavior of polyamides is becoming a topical issue because of their new industrial application domains. Substantial research is thus currently ongoing to gain further knowledge on mechanisms affecting the fatigue lifespan, which is often limited by strain concentration and followed more or less quickly by crack inception. Irreversible fatigue mechanisms are associated with dissipation of part of the deformation energy rate, with the complementary part being associated with - at least temporarily - stored energy variations induced by microstructural changes. A promising approach to better monitor and gain insight into these 
mechanisms is based on the use of quantitative infrared thermography and optical extensometry. Infrared and visible analysis techniques have been successfully used for several years to characterize and understand the behavior of materials (Grediac and Hild, 2012). Dissipation assessment has been the focus of numerous surveys over the last century (Bever et al., 1972). Several experimental approaches based on temperature measurements via quantitative IR techniques have recently been adopted to estimate this crucial energy signature (Chrysochoos and Barton, 2015). Indeed, dissipation can be derived from thermal data fields by estimating the partial differential operators of the heat diffusion equation (Chrysochoos et al., 1989). Several assumptions on thermal fields and dissipation distributions have been formulated to make heat diffusion models operational. A number of simplified formulations are now available for dissipation assessments (Chrysochoos and Louche, 2000).

As already mentioned, PAs are not only sensitive to their thermal state but are also hydrophilic materials inasmuch as their water content strongly influences their mechanical behavior. It is thus essential to detect the existence of so-called water plasticizing effects when using PAs for specific industrial applications where the environmental humidity changes considerably. Calorimetric signatures of these effects can also be provided and, consequently, contribute to the understanding of the material behavior. Indeed, water plasticizing effects are recognized for their marked influence on the glass transition temperature of some PAs (Brandrup et al. 1999). Glass transition can be defined as a gradual thermally activated process that leads a polymer to change from a glassy to rubbery state (Sperling, 1992; Rodriguez, 1996). The addition of water molecules to the polymer microstructure causes a decrease in the glass transition temperature as, for instance, recently reported for PA6.6 in (Launay et al., 2013). The glass transition temperature markedly decreases as the relative humidity increases, which means that PA6.6 shows rubber effects under standard usage temperatures. Several approaches have been developed in the past to describe the thermomechanical rubber (entropic) effects and so-called thermoelastic inversion. Here we ought to mention the pioneering studies of Joule and Treloar (Joule, 1857; Treloar, 1973; Treloar, 1975). Many other major studies were thereafter conducted, e.g. Flory and Erman (1973), Chadwick and Creasy (1984), Arruda and Boyce (1993), etc. More recently, a simple heuristic description of thermoelastic inversion in rubber materials was proposed in Benaarbia et al. (2014b). This inversion was interpreted as being due to competition between standard and entropic thermoelasticity.

In the present paper, we documented some findings pertaining to the local thermomechanical mechanisms of the polyamide-6.6 matrix. Specifically, we used IR and visible CCD cameras to simultaneously record, during cyclic loadings, fields corresponding to temperature variations and in-plane displacements over the sample gage part. We focused particularly on local estimation of dissipation, coupling sources and strain rate maps throughout the cyclic test in order to correlate thermal, energy and mechanical signatures. This analysis enabled us to characterize the onset and development of dissipative strain concentration zones, which could ultimately be related to the local failure of the studied material.

Prior to that, we first reminded the theoretical framework used to define the energy balance form and interpret the cyclic thermomechanical responses of wet PA6.6. We then presented a very brief overview of overall observations made on PA6.6 specimens, namely material responses averaged over the sample gage part.

\section{Thermomechanical framework}


In the framework of the thermodynamics of irreversible processes with internal state variables, the equilibrium state of each volume material element is characterized by a set of $n$ state variables (Germain et al., 1983). Generally speaking, for finite transformations, the often chosen state variables are the absolute temperature $T$, the Hencky strain tensor $\varepsilon$, and the $n-2$ scalar components $\left(\alpha_{1}, \ldots, \alpha_{n-2}\right)$ defining a vector $\alpha$ of internal variables, introduced to depict the micro-structural state of the material. By construction, the thermodynamic potential is the specific Helmholtz free energy $\psi$.

The reversible part of the Cauchy stress tensor $\sigma$ is the conjugated variable associated with the strain with respect to the free energy and is defined by $\sigma^{r}=\rho \partial \psi / \partial \varepsilon=\rho \psi,{ }_{\varepsilon}, \rho$ denoting the mass density. For finite strain, the volume deformation energy rate can then be written as:

$$
w_{\text {def }}^{\bullet}=\sigma: D=\underbrace{\sigma: D-(\overbrace{\sigma^{r}: \dot{\varepsilon}-\rho \psi_{, \alpha} \cdot \dot{\alpha}}^{w_{e}^{*}+w_{\dot{s}}^{*}})}_{\mathcal{D}_{1}}+w_{\dot{e}}^{\bullet}+w_{s}^{\bullet} \text {, }
$$

where $D$ is the Eulerian strain rate tensor. According to the material and the loading path, the deformation energy rate $w_{d e f}^{\bullet}$ may involve energy dissipation $\left(\mathcal{D}_{1}=\sigma: D-\sigma^{r}: \dot{\varepsilon}-\rho \psi_{\alpha_{\alpha}} . \dot{\alpha}\right)$, elastic $w_{e}^{\bullet}$ and stored/released $w_{s}^{\bullet}$ energies. The intrinsic dissipation is the heat rate induced by the irreversibility of the material transformation while the elastic and stored energy rates depict respectively a deformation energy mechanically recoverable during unloading and used in the microstructural changes. Note that the non-standard notation $w^{\bullet}$ specifies that the energy rate is path-dependent (i.e. $w$ is not necessarily a state function).

Combining the local expressions of the first and second principles of thermodynamics leads to the local heat diffusion equation:

$\rho C \dot{T}-\operatorname{div}(\operatorname{kgradT})=\mathcal{D}_{1}+\underbrace{\rho T \psi_{,_{T \varepsilon}}: \dot{\varepsilon}+\rho T \psi_{, T \alpha} \cdot \dot{\alpha}}_{w_{t h c}^{*}}+r_{e}$

where $C$ is the specific heat, $k$ is the heat conduction tensor. The left-hand side consists of a differential operator applied to the temperature, while the right-hand side pools the various types of heat sources: the so-called intrinsic dissipation $\mathcal{D}_{1}$, the thermo-mechanical coupling sources $w_{t h c}^{\bullet}$, and the possible external heat supply $r_{e}$ (e.g. radiation exchanges). The thermomechanical heat sources give effects to the thermo-sensitivity of the matter, indicating that the mechanical, microstructural and thermal states are closely coupled. In reference to what we previously evoked in introduction, note that a hygrometric variable should be introduced in the set of the microstructural state variables to take the water plasticizing effects into account.

Let us now consider a load-unload cycle, where $A=\left(T_{A}, \varepsilon_{A}, \alpha_{A}\right)$ and $B=\left(T_{B}, \varepsilon_{B}, \alpha_{B}\right)$ denote the thermodynamic states of the material at the extremities of the process. Both principles of thermodynamics give an alternative expression of the volume deformation energy associated with the load-unload test:

$w_{d e f}=\int_{t_{A}}^{t_{B}} \mathcal{D}_{1} d \tau+\int_{t_{A}}^{t_{B}}(\rho \dot{e}-\rho C \dot{T}) d \tau+\int_{t_{A}}^{t_{B}} w_{t h c}^{\cdot} d \tau$

where $e$ is the specific internal energy and $t_{B}-t_{A}$ is the cycle duration. 
- In the general case $(A \neq B)$, the balance of deformation energy during a load unload test involves energy dissipation, internal energy variations, variation of heat stored in the material and coupling heat sources.

- If now $\left(\varepsilon_{A}=\varepsilon_{B}\right)$, the stress-strain diagram shows a hysteresis loop. The deformation energy per cycle then corresponds to the "generalized area" $A_{h}$ of the different hysteresis loops in the planes $\sigma_{i j}-\varepsilon_{i j}, i, j=1,2,3$. This hysteresis area depicts a loss of mechanical energy that can be, as previously mentioned, potentially induced by energy dissipation, stored energy variations and/or strong thermo-mechanical coupling effects.

- Finally if $(A=B)$, the mechanical cycle is now a thermodynamic cycle. The hysteresis loop is only due to dissipation and couplings since the heat capacity per unit volume $\rho C$ is assumed to be constant.

It is worth noting that the existence of an infinite lifespan of materials subjected to cyclic loading is possible when there is no intrinsic dissipation (i.e. no material irreversibility) or, conversely, when the hysteresis area is all intrinsically dissipated. In this last very particular case, however often mentioned in the literature, the mechanical cycle being a thermodynamic cycle, the material should periodically pass through the same thermodynamic state $A$ and an infinite lifespan should then be ensured. For finite lifespan, intrinsic dissipation and internal energy variations, depicting the gradual evolution of the microstructure, accompany the irreversible fatigue mechanisms.

\section{Overall analysis}

Many experimental results dealing with the thermomechanical cyclic behavior of PA6.6 matrix have been summarized and thoroughly commented in previous review papers (Benaarbia et al., 2014a,b). In particular, the kinetics of hysteretic responses involved during the fatigue of PA6.6 specimens have been closely analyzed in Benaarbia et al. (2014b).

\subsection{Mechanical analysis}

One of the goals of this first series of experimental tests was to check if stabilized cyclic behavior could be observed from mechanical (corresponding to the case $\left(\varepsilon_{A}=\varepsilon_{B}\right)$ of the last section) and/or thermodynamic (corresponding to the case $(A=B))$ standpoints. Indeed, the existence of a stabilized cyclic "state" is often pointed out in the literature and plays a key role in the construction of fatigue lifespan criteria (Blanche et al., 2014). In what follows, we focused particularly on the beginning of the cyclic test where we observed that the overall material responses changed drastically. We also opted to consider the symptomatic case of PA6.6 with a relative humidity $(\mathrm{RH})$ of about $80 \%$ and with the cyclic test being performed at a loading frequency $f_{L}$ of $1 \mathrm{~Hz}$ for a stress ratio $R_{\sigma}$ of 0.1 and a stress range $\Delta \sigma$ of $36 \mathrm{MPa}$.

Three parameters were introduced to characterize variations in hysteresis loops, namely: $i$ ) the hysteresis area, which represents the deformation energy lost within a loading cycle, ii) the mean slope of the hysteresis loop, which can be interpreted as overall structure stiffness, and iii) the mean strain over a cycle, which reflects the creep induced by a nonzero mean stress (see. Fig.1). The mechanical results showed that the hysteresis area decreased markedly at high relative humidity and low strain rates. The mean slope was also observed to decrease markedly 
at the beginning of the tests and seemed to stabilize but never before a few hundred cycles. Besides, it was found that the cycle by cycle shifting of hysteresis loops (i.e. ratcheting) was more pronounced for wet specimens, and that the cyclic stabilization of the three parameters never occurred simultaneously (see. Fig. 1-(b)).

(a)

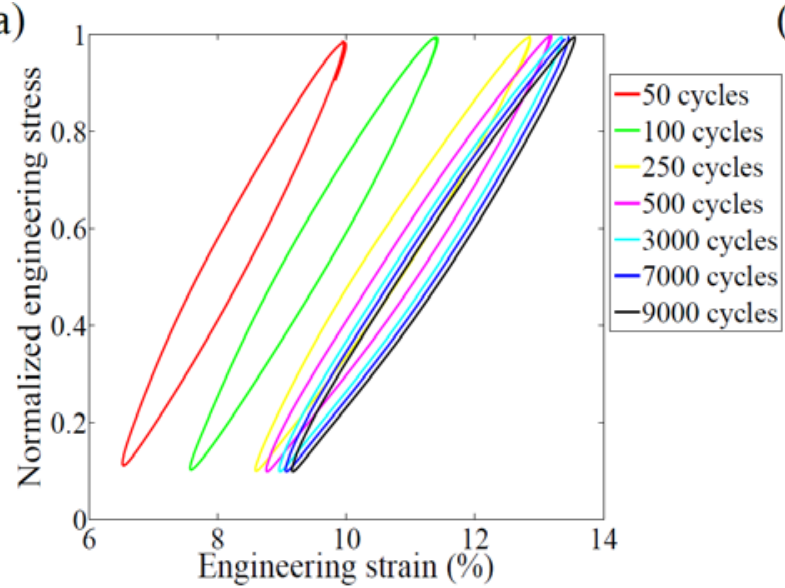

(b)

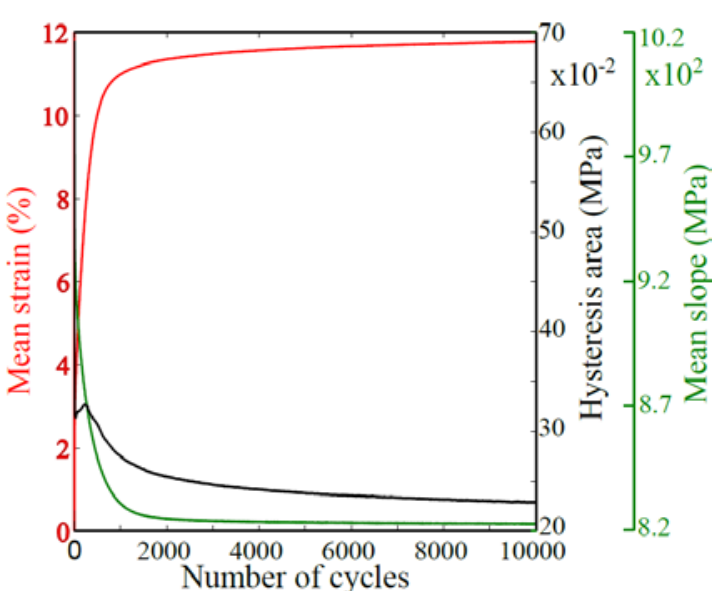

Fig. 1: (a) Normalized engineering stress versus engineering strain curves showing shifting of the mechanical hysteresis loops. (b) Hysteresis area, mean slope and mean tensile strain per cycle as a function of the number of cycles. The loading frequency $f_{L}=1 \mathrm{~Hz}$ and the stress ratio $R_{\sigma}=0.1$.

\subsection{Thermal analysis}

From a thermal standpoint and for low loading rates (here $f_{\mathrm{L}}=1 \mathrm{~Hz}$ ), it was noted that temperature variations increased rapidly at the very beginning of the cyclic loading, reaching a maximum and then decreased gradually after several hundred cycles (see. Fig. 2, dark plot). This self-heating at the beginning of the cyclic test was naturally compared to the rapid + changes in the three previous parameters characterizing the cyclic evolution of the hysteresis loop. It was noted that the temperature decrease could be related to a decrease in the dissipation intensity cumulated with heat exchanges between the specimen and the surroundings (see. Fig. 2, red plot).

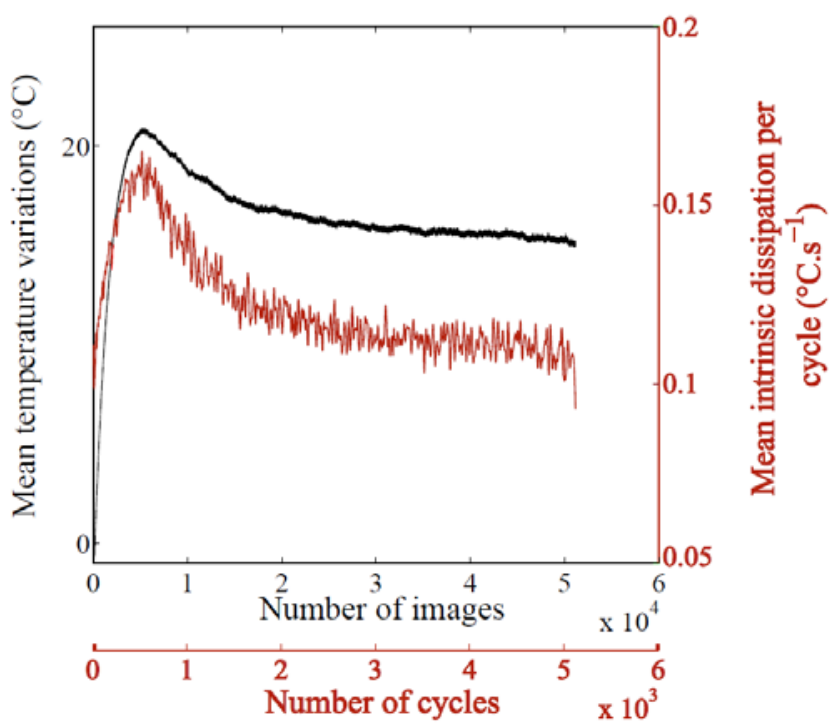

Fig. 2: Time course of temperature variations and mean intrinsic dissipation per cycle associated with a cyclic test performed on wet PA6.6. 


\subsection{Energy analysis}

From a calorimetric standpoint, it was found that temperature variations were induced by two types of heat source: dissipative and coupling sources. Note that the intrinsic dissipation, being the calorimetric signature of material irreversibilities, is non-negative while thermomechanical coupling sources change sign at each loading inversion. Two coupling effects were detected: i) standard thermoelastic source which reflects the thermodilatability of the material and can be detected via symptomatic thermal oscillations out of phase - under adiabatic conditions - with the monochromatic loading signal, ii) rubber (also termed entropic) elastic source, related to the stress-induced orientation/disorientation of macromolecular chains, leading to temperature oscillation in phase with the monochromatic loading signal. Both sources match with the coupling terms introduced in Eq. (2):

$w_{t h c}^{\bullet}=w_{t h e}^{\bullet}+w_{r}^{\bullet}=\rho T \psi_{,_{T \varepsilon}}: \dot{\varepsilon}+\rho T \psi_{,_{T \alpha}} \cdot \dot{\alpha}$

It is worth noting that the standard thermoelastic source $w_{\text {the }}^{\bullet}$ and the rubber thermoelastic source $w_{r}^{\bullet}$ do not correspond respectively and systematically to the coupling terms $\rho T \psi_{, T \varepsilon}: \dot{\varepsilon}$ and $\rho T \psi_{, T \alpha} . \dot{\alpha}$. The correspondence depends on the chosen set of state variables.

As mentioned above, the coexistence of standard and entropic thermoelasticity led to the famous thermoelastic inversion, the entropic term being prevalent at finite strain.

In Benaarbia et al., 2014(b,c), we recently modeled this competition using the Generalized Standard Material formalism (Halphen and Nguyen 1975). The challenge was to be in accordance with the statistical formulation of the rubber elasticity while also being in position to use the GSM approach to formulate the constitutive equations of PA6.6.

We proposed a 1D heuristic approach of the thermoelastic inversion, based on the ideal gas analogy formulated by Treloar 1975: the internal energy $e_{r}$ of an entropic elastic (rubber-like) material is not strain-dependent and is solely a function of the absolute temperature $e_{r}(s, \varepsilon)=$ $\tilde{e}(T)$, (i.e. a typical characteristic of an ideal gas).

The part of the PA6.6 free energy dedicated to thermoelastic couplings was finally seen as the sum of the free energy $\psi_{e}$ defined for a standard thermoelastic material and that of a rubber material $\psi_{r}$. Then, when considering the Hencky (logarithmic) strain, we supposed that the total strain tensor could be split into an elastic part $\varepsilon_{e}$ and rubber part $\varepsilon_{r}$, as is the case for any series model. For the 1D model with incompressible rubber transformation, the overall specific thermoelastic free energy $\psi_{\text {the }}$ could then be written as:

$$
\psi_{\text {the }}\left(\theta, \varepsilon, \varepsilon_{r}\right)=\underbrace{\frac{1}{2} E\left(\varepsilon-\varepsilon_{r}-\alpha_{t h} \theta\right)^{2}-\overbrace{\frac{1}{2}\left(\frac{\rho C}{T_{0}}+E \alpha_{t h}^{2}\right) \theta^{2}}^{\psi_{r}}+\mathcal{K}_{r}\left(\theta+T_{0}\right)\left(\frac{e^{2 \varepsilon_{r}}}{2}+e^{-\varepsilon_{r}}-\frac{3}{2}\right)}_{\psi_{e}})^{(5)}
$$

where $E$ stands for the elastic modulus, $\alpha_{t h}$ the thermal expansion coefficient, $\theta=T-T_{0}$ the change in the current temperature $T, T_{0}$ representing the room temperature and $\mathcal{K}_{r}$ a material constant.

In the agreement with the foregoing, the coupling sources could thus be derived from Eq. (5). With the simplified 1D formulation, Eq. (4) could be rewritten as:

$$
w_{t h c}^{\bullet}=w_{t h e}^{\bullet}+w_{r}^{\bullet}=-E \alpha_{t h} T\left(\dot{\varepsilon}-\dot{\varepsilon}_{r}\right)+\mathcal{K}_{r} T\left(e^{2 \varepsilon_{r}}-e^{-\varepsilon_{r}}\right) \dot{\varepsilon}_{r}
$$


In agreement with observations, Eq. (6) first showed that the standard thermoelastic source was predominant as long as the rubber strain remained negligible compared with the overall strain. The overall thermoelastic source was then negative during a tensile loading. An order of magnitude of the standard thermoelastic strain $\dot{\varepsilon}-\dot{\varepsilon}_{r}$ can be provided about $\sigma / E \approx 0.01$. So, since the overall strain was about 0.1 , the rubber effects were prevalent and the overall heat source became positive. The strain at which both sources had the same order of magnitude corresponded to the so-called thermoelastic inversion (Anthony et al., 1942).

Besides, as long as the temperature variations remained small with respect to the absolute temperature $\left(T \approx T_{0}\right)$, Eq. (6) still showed that the mean coupling sources averaged over a complete mechanical cycle remained negligible:

$$
\lim _{\substack{T(\tau) \rightarrow T_{0} \\ \forall \tau \in\left[t, t+\frac{1}{f_{L}}\right]}}\left\langle\int_{t}^{t+\frac{1}{f_{L}}} w_{t h c}^{\bullet} d \tau\right\rangle \approx 0
$$

In practice, for PA6.6, this mean energy induced by couplings remained low compared to the size of the hysteresis loop. This naturally does not mean that the corresponding energy rates were negligible. On the contrary, the range of the thermoelastic coupling sources may become even more than several tenfold greater than the mean dissipation intensities per cycle.

Finally, from a more general energy standpoint, the mean stored energy rate per cycle was computed as the difference between the mean deformation rate and the mean dissipated energy rate per cycle, the mean elastic energy rate vanishing over a complete loading cycle (Eq.(1)). We observed, in each case, a non-negligible change in the stored energy, which meant that the mechanical hysteresis loop could never be associated with a thermodynamic cycle even though the loop quasi-stabilized in some cases.

We again stress that the foregoing findings were obtained from a standard overall analysis of the thermomechanical data extracted from cyclic tensile tests, while implicitly assuming that the gage part of the specimen behaved homogeneously. This crucial hypothesis naturally reduced the data processing computation time and allowed us to study complete cyclic tests containing up to $2.10^{6}$ images under various loading conditions (frequency, humidity level, stress ratio, stress range). However, we claimed that investigations into the development of possible heterogeneities had to be conducted, particularly at the beginning of the cyclic test where all parameters describing the global thermomechanical behavior drastically changed. The goal of what follows was then to track the potential onset of strain concentration mechanisms.

We therefore combined imaging techniques (i.e. infrared thermography (IR) and digital image correlation (DIC)) to assess the creep and cyclic strain fields and also to obtain further information on the associated dissipative and coupling mechanisms. In the sequel, a brief presentation of the experimental setup and imaging techniques was proposed. For readers interested in optical metrology and image processing techniques, more details and references were given in appendix.

\section{Experimental setup and methodology}

The experimental setup involved an MTS 810 hydraulic testing machine equipped with a load cell of $\pm 25 \mathrm{kN}$, a high resolution infrared focal plane array camera and a high resolution visible 


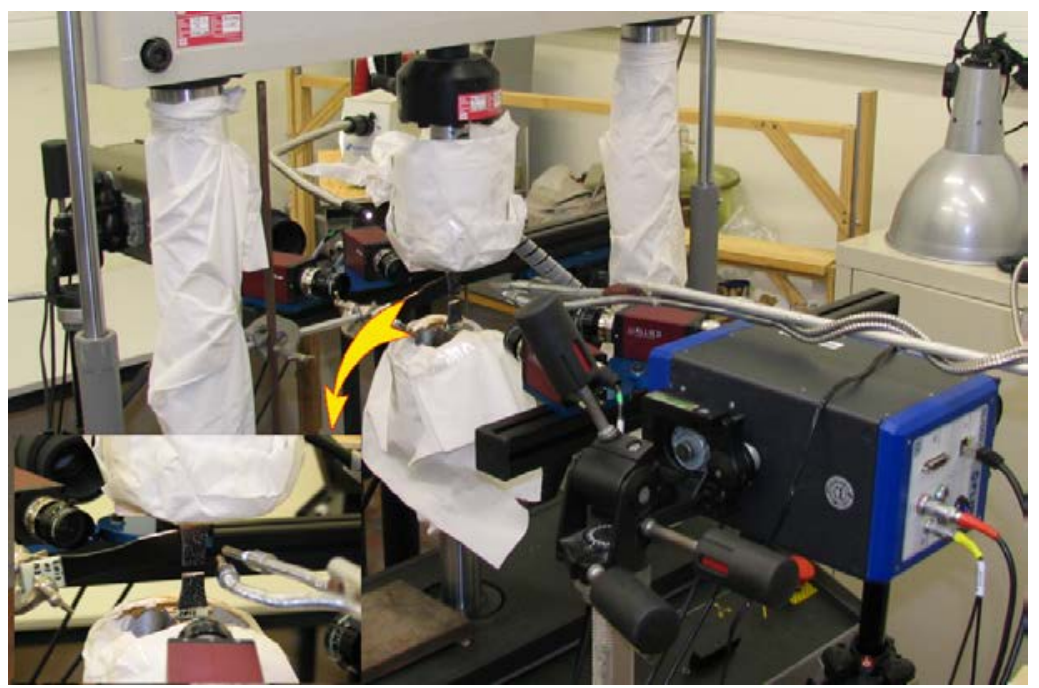

Fig. 3: Experimental setup showing both cameras used for recording the displacement and temperature fields.

In this work, the thermal measurements were conducted using an IR Flir Titanium camera. This was fitted with an InSb matrix detector, which is sensitive to infrared radiation in the 3-5 $\mu \mathrm{m}$ wavelength range (MW middle waves). The camera was equipped with a $25 \mathrm{~mm}$ lens and positioned on a tripod approximately $0.5 \mathrm{~m}$ from the specimen surface. The detector was operated with a 320 x 256 pixel window size and $29 \mu \mathrm{m}$ pixel size. The camera frame rate was set at tenfold the loading frequency to obtain enough sampled data per cycle, and thus to improve — via fitting techniques - the signal-to-noise ratio. We also decided to work with a $400 \mu$ s integration time for a temperature range of $\left[15^{\circ} \mathrm{C}, 48^{\circ} \mathrm{C}\right]$. The test was performed under ambient conditions with a room temperature of about $T_{r}=23^{\circ} \mathrm{C}$. It was necessarily to implement an adequate calibration procedure to reduce the measurement error. This calibration method was performed for each pixel of the element matrix (Honorat et al., 2005). This pixel calibration protocol was based on a polynomial fitting of the digital levels delivered by each individual detector element. Regarding the material emissivity, this emission ratio was raised and homogenized by coating the test sample with matt black paint, so it was thus assumed that the sample was perfect black body with an emissivity value close to 1. Moreover, other experimental precautions were taken into account in order to overcome difficulties in thermal measurements. Readers interested in the tricky metrological aspects of infrared thermography techniques are referred to Berthel (2007), Benaarbia (2014d), etc.

The kinematic data were recorded using a Phantom V12 high speed camera with a $20 \mu \mathrm{m}$ pixel size. The detector was operated with 256x256 pixels due to the hard drive constraints with the computing resources available in the lab. This relatively low resolution limited the spatial precision of the visible measurements, but allowed high frame rates. In this work, we also decided to work with a $10 \mathrm{~Hz}$ acquisition frequency and $1200 \mu$ s integration time. The random pattern (local optical signature of a material surface element) was achieved by spraying the specimen surface with a painted speckle pattern (pulverization of black and white paints). The camera required an intensive light source in order to provide uniform lighting and thus clearly record visible images at this acquisition frequency. To accommodate this, a cold light source was clamped around the test sample, which lit the targeted region. Adequate DIC software was required to obtain information on positional changes on the surface under investigation. In our 
case, we used the tailored homemade Kelkins software package. Displacement fields were thus determined after digitizing images captured by the visible camera.

The geometric transformation between the frames of reference of the two cameras was determined by using a calibrated target. The correspondence between the spatial coordinates of the thermal image $\left(x_{T}, y_{T}\right)$ and those of the visible image $\left(x_{K}, y_{K}\right)$ was established by triggering the motion of the calibrated target using the following equations:

$$
\left(\begin{array}{l}
x_{K} \\
y_{K}
\end{array}\right)=\underbrace{\left(\begin{array}{ll}
a_{x} & b_{x} \\
a_{y} & b_{y}
\end{array}\right)}_{\boldsymbol{R}}\left(\begin{array}{l}
x_{T} \\
y_{T}
\end{array}\right)+\underbrace{\left(\begin{array}{l}
c_{x} \\
c_{y}
\end{array}\right)}_{\boldsymbol{d}},
$$

where $\boldsymbol{R}$ is the rotation matrix and $\boldsymbol{d}$ a displacement vector. This correspondence procedure was carried out for each cycle of the test.

Finally, the temporal synchronization of the IR and visible cameras was achieved using the load cell sensor of the hydraulic testing machine (MTS). A specific tailored electronic device called Synchrocam was designed to capture both kinematic and thermal data simultaneously. Both imaging systems were then triggered from a digital pulse to capture data concurrently. In the current investigation, both systems were initiated at the same time with identical frame rates, which facilitated the image processing.

\section{Experimental results}

The PA6.6 samples were provided by Solvay Engineering Plastics (cf. Fig.4). Tensile specimens of $100 \mathrm{~mm}$ in overall length by $20 \mathrm{~mm}$ in gage width and $3.14 \mathrm{~mm}$ in thickness were cut from plates using a high speed router. They were conditioned in an environmental chamber under $80 \%$ relative humidity $(\mathrm{RH}=80 \%)$. The hygrothermal state was reached by conditioning the samples at $65^{\circ} \mathrm{C}$ and then at $35^{\circ} \mathrm{C}$ until mass stability. The samples were regularly weighed to ensure that water equilibrium was reached. The conditioning period was around 40 days. After conditioning, the samples were placed in sealed envelopes to keep a constant humidity until the mechanical test. Just before the test, the specimens were extracted from the envelope and quickly placed in the hydraulic grips of the testing machine. The duration of the test was chosen sufficiently short compared to water diffusion characteristic time to admit that the humidity remained constant throughout the fatigue test.

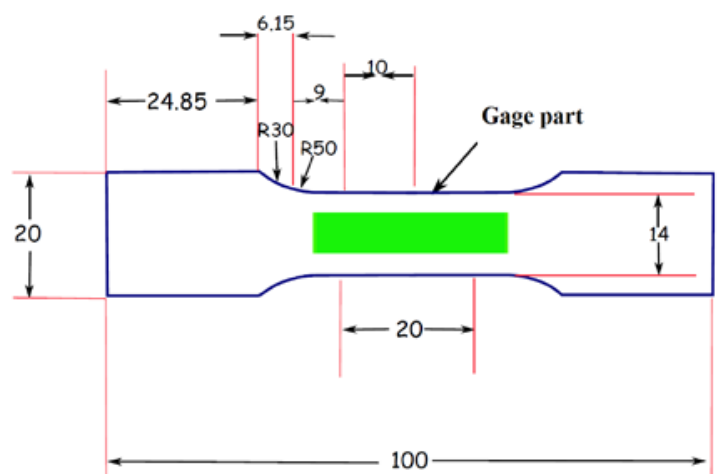

Fig. 4: Basic sketch of specimens used for experiments (all dimensions are in millimeters).

Cyclic tensile-tensile loading was performed at $1 \mathrm{~Hz}$ loading frequency and 0.1 stress ratio, while the number of cycles was set at 10000 cycles. This number of cycles was estimated to be high enough to check for the possible existence of a stabilized cyclic behavior in PA6.6. At the beginning of the test, the load signal was set at zero for $10 \mathrm{~s}$ in an attempt to capture a reference 
thermal image. Thereafter the displacement rate was controlled with a constant cross-head velocity of $1 \mathrm{~mm} . \mathrm{s}^{-1}$, and was then held at $\sigma_{\min }$ for $3 \mathrm{~s}$. This first part of the loading path was then referred to as stage 1 . A sinusoidal signal was then applied between $\sigma_{\min }$ and $\sigma_{\max }$ throughout the cyclic test. We called this second part of the mechanical load stage 2.

We then investigated the kinetics of the strain rate fields in relation to the dissipation distribution. In particular, we proposed to compare the dissipative contribution of the creep (ratcheting) and cyclic effects. We also correlated the spatial heterogeneities of kinematic and calorimetric fields. The aim was to check if a kind of cyclic stabilization could be locally detected. Finally, we investigated the distribution of the thermoelastic source ranges and the thermoelastic inversion effects.

\subsection{Kinematic analysis}

\subsubsection{Preloading until $\sigma_{\min }$}

Fig.5-(a) represents the mechanical loading path used in the experiments. The boundary between stage 1 and stage 2 was established at the "start" pink point. As already mentioned, DIC techniques were systematically used to derive the different kinematic variables. The kinematic image size was roughly equivalent to a box of $35 \mathrm{~mm}$ in length by $7.35 \mathrm{~mm}$ in width, covering approximately $52 \%$ of the gage specimen surface. The spatial resolution for the kinematic data was set at $r_{\mathrm{s}}=0.35 \mathrm{~mm} / \mathrm{pixel}$. The smoothing parameters used for the postprocessing of the experimental data were: $N_{x_{K}}=10, N_{y_{K}}=4$ and $N_{t}=10$ (see Appendix).

In Fig. 5-(b), the longitudinal Hencky strain field $\bar{\varepsilon}_{x x}$ was computed at time $t_{\text {start }}$ (see Appendix for more information on the strain assessments). In Fig.5-(c), the associated longitudinal profile, assessed by averaging the 2D strain distribution over the sample width, was plotted. First, the high pre-deformation during the preloading should be mentioned. During stage 1, the specimen strongly deformed up to approximately $7 \%$, even before starting the cyclic loading (stage 2). This noteworthy result highlighted the high ductility of PA6.6. Moreover, the strain data conspicuously indicated some strain peak focused at the center of the gage part. A zoomed profile in Fig 5-(c) is superimposed to highlight the heterogeneous distribution of the longitudinal strain components along the sample gage length. The standard deviation value corresponding to these spatial heterogeneities was approximately 20 -fold lower than the mean strain value. This ratio gave an idea of the intensity of the strain pattern heterogeneity.

(a)

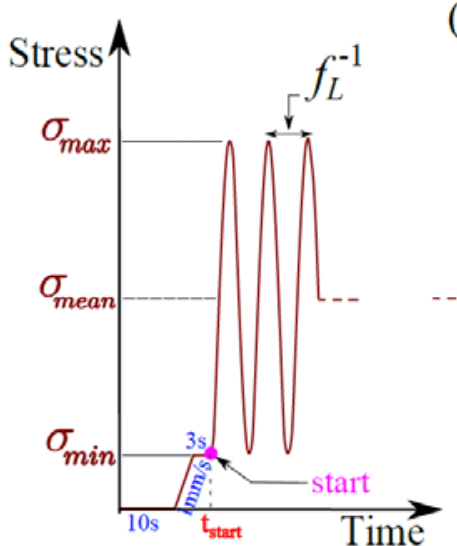

(b)

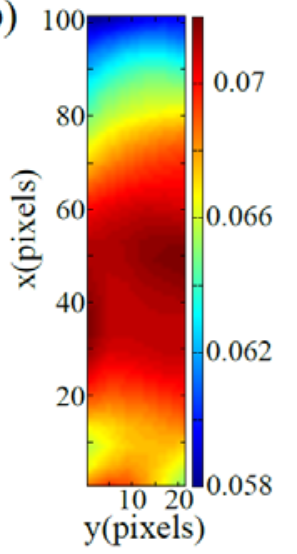

(c)

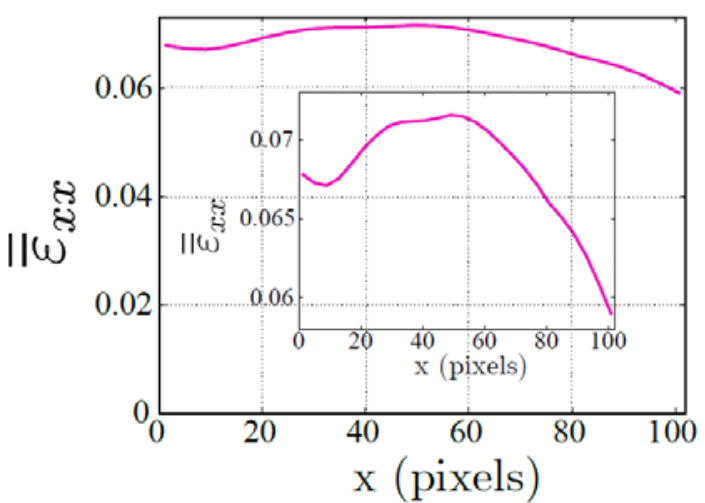

Fig. 5: (a) Diagram of the mechanical preloading used in the experiments. (b) 2D map of the pre-existing longitudinal strain component at time $t_{\text {start, }}$ and (c) corresponding width-wise 
averaged profiles $\overline{\bar{\varepsilon}}_{x x}$ as a function of the $x$ coordinate. The inner diagram is a simple zoom of the outer one.

The origins of this initial heterogeneous and dissymmetric strain distribution were currently hard to precisely identify. We estimated that the causes were of material origins and did not come from an artifact induced by a slip of the specimen heads in the hydraulic grips inducing a complex loading path. Indeed, we did not observed first any sliding mark induced by the jaws on the specimen's heads, and second, the DIC assessments, being totally decoupled from the testing machine and its grip system, allowed us to check that the loading conditions was that of a cyclic simple tension test. We therefore temporarily associated this systematic initial heterogeneous, dissymmetric response with a material heterogeneity probably induced by the injection and machining processes and amplified by a non-perfectly uniform distribution of water content.

\subsubsection{Maps and profiles of the longitudinal strain component}

The second kinematic assessments were done in terms of the mean Hencky strain per cycle. Fig. 6 depicts the spatial distributions of the mean longitudinal strain component per cycle $\tilde{\bar{\varepsilon}}_{x x}$ captured at different fatigue stages. The strain patterns shown in Fig. 6 indicated a precocious and gradual development of spatial strain concentration zones. These spatial heterogeneities developed preferentially close to the center of the gage length where the strain peak initiated during preloading. The zone spread rapidly until it covered the entire gage width and spread in the length direction. The statistics associated with these strain distributions are pooled in Table 1. Note that, at the very beginning of the loading, the standard deviation values (SDVs) corresponding to these spatial distributions were approximately 24-fold lower than those of the mean values. The standard deviation increased considerably until the $500^{\text {th }}$ cycle, then stayed quasi-constant after the $1000^{\text {th }}$ cycle, whereas the mean values grew continuously throughout the loading. This means that, at the very beginning of the loading, there was obvious development of spatial heterogeneity, but this heterogeneity "quasi-stabilized" after the $1000^{\text {th }}$ cycle, in the sense that the ratio of the standard deviation over the mean value of the strain profiles remained roughly constant afterwards. Furthermore, the associated average width-wise profiles are plotted in Fig. 7. These profiles indicated a marked increase in the mean longitudinal strain per cycle, especially near the $53^{\text {th }}$ pixel in length. Again the strain pattern time course can be divided into two major periods. The first one was characterized by a steep increase during the first few thousands of cycles, and it lasted 500 cycles, whereas the second period was characterized by a lower increase and lasted throughout the rest of the fatigue test. These results are consistent with the ratcheting effects observed in Fig. 7-(a,b). The hysteresis loop shifted rapidly at the beginning of the cyclic loading and progressively slowed down without ever completely stabilizing. These noticeable observations are replied in the next subsections and compared with other kinematic of calorimetric manifestations.

Table 1: Standard deviation and spatial averages associated with the strain maps obtained in Fig. 6.

\begin{tabular}{|l|c|c|c|c|c|c|c|c|}
\hline & $\begin{array}{c}50 \\
\text { cycles }\end{array}$ & $\begin{array}{c}100 \\
\text { cycles }\end{array}$ & $\begin{array}{c}250 \\
\text { cycles }\end{array}$ & $\begin{array}{c}500 \\
\text { cycles }\end{array}$ & $\begin{array}{c}1000 \\
\text { cycles }\end{array}$ & $\begin{array}{c}3000 \\
\text { cycles }\end{array}$ & $\begin{array}{c}5000 \\
\text { cycles }\end{array}$ & $\begin{array}{c}9000 \\
\text { cycles }\end{array}$ \\
\hline$S D \times 10^{-3}$ & 3.72 & 3.70 & 5.62 & 9.32 & 9.41 & 9.45 & 9.51 & 9.53 \\
\hline Average $\times 10^{-2}$ & 8.91 & 9.31 & 9.81 & 10.71 & 11.01 & 11.11 & 11.28 & 11.33 \\
\hline
\end{tabular}



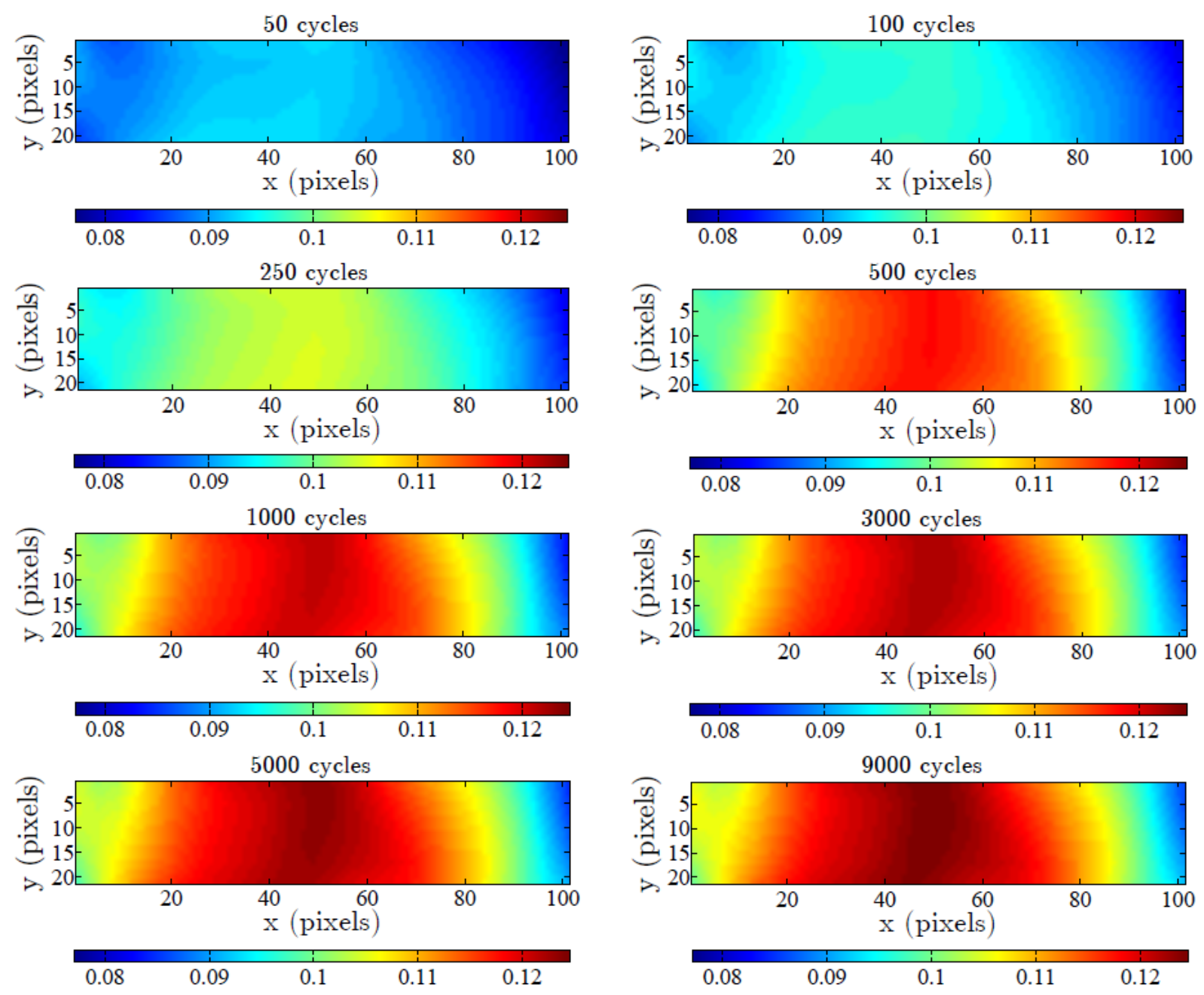

Fig. 6: $2 D$ fields of the mean longitudinal strain component per cycle captured at different fatigue stages in the wet PA6.6 matrix.

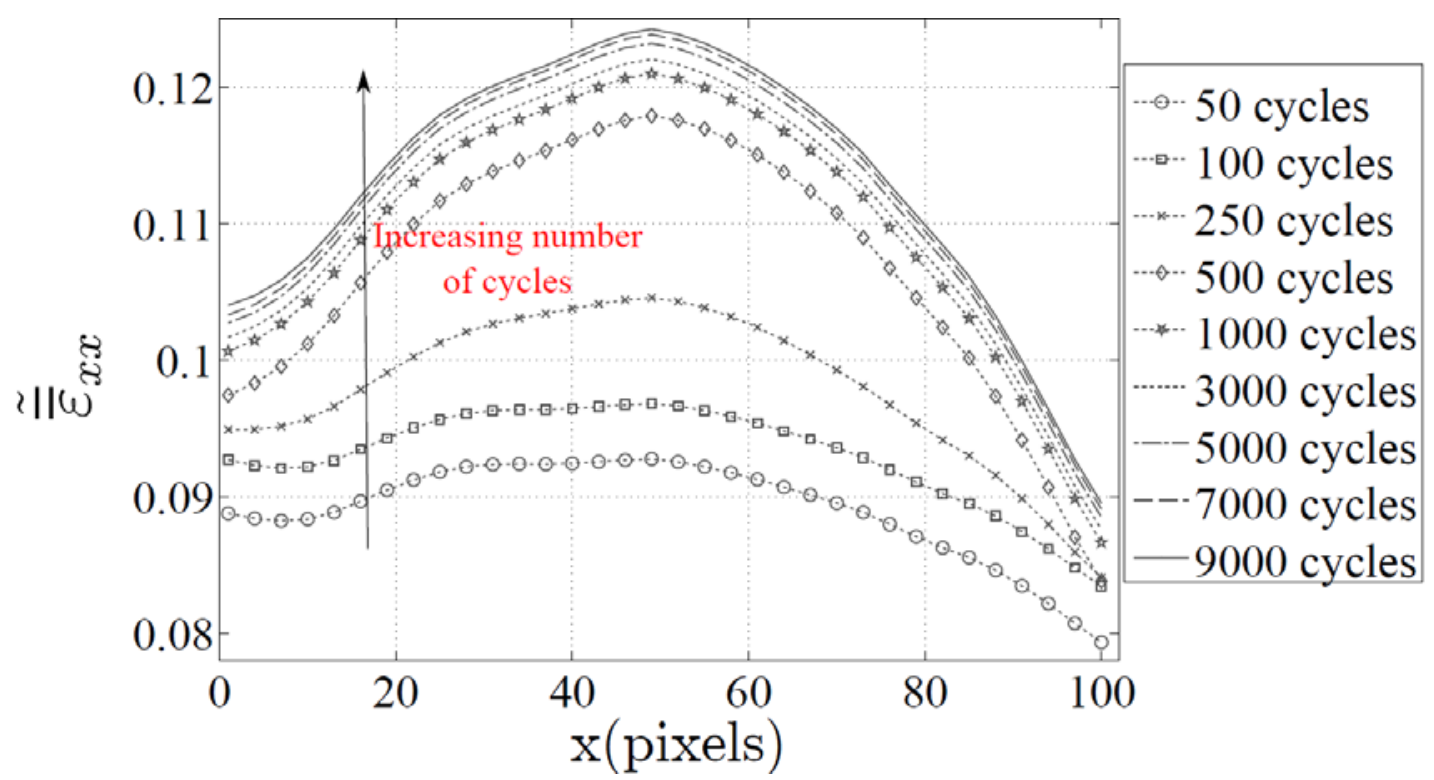

Fig. 7: Average width-wise profiles of the longitudinal strain component per cycle as a function of the $\mathrm{x}$ coordinate associated with the selected cycles. 


\subsubsection{Maps and profiles of the equivalent strain rates}

The cyclic deformation effects of PA6.6 can be formally split into two mechanisms: the oscillatory strain response due to cyclic loading and the monotone creep response induced by the mean stress, with the stress ratio being kept at $R_{\sigma}=0.1$.

We then used the general rheological framework of viscosity to describe the irreversible character of the deformation process. Plasticity concepts were not used because nor threshold effects nor time independent behavior were until now identified.

For simplicity reasons, we then conjectured an additive partition of the strain effects. We associated the oscillatory strain response to thermoelastic and viscoelastic effects, the rubber effects and the recoverable part of the creep (recovery effects) being related to a viscoelastic rubbery strain $\bar{\varepsilon}_{r}$. The non-recoverable part of the creep response was supposed to be described by a pure viscous strain $\bar{\varepsilon}_{v}$. We then supposed that:

$\bar{\varepsilon}=\bar{\varepsilon}_{e}+\bar{\varepsilon}_{r}+\bar{\varepsilon}_{v}$

The rate of the two first strain contributions changed sign during each loading inversion while the non-recoverable part of the creep strain rate remained always positive because of the positive mean stress. We finally considered that (i) the elastic energy associated with the rubber strain was (at least temporarily) stored, and (ii) the viscoelastic and non-recoverable creep strains were accompanied by energy dissipation.

We therefore defined two types of strain rate:

- An equivalent strain rate averaged over a complete cycle $\tilde{\bar{\varepsilon}}_{e q}$, named the “cyclic strain rate”, was conventionally computed as:

$\tilde{\overline{\bar{\varepsilon}}}_{e q}=\int_{n f_{L}^{-1}}^{(n+1) f_{L}^{-1}} \sqrt{\dot{\bar{\varepsilon}}_{x x}^{2}+2 \dot{\bar{\varepsilon}}_{x y}^{2}+\dot{\bar{\varepsilon}}_{y y}^{2}}$

where $n$ stands for the cycle number.

Note that Eq. (10) involved only experimentally available in-plane strain rate components. That's the reason why no particular normalization coefficient was introduced in Eq. (10) as it is usual for standard equivalent strain rates.

Moreover from a quantitative standpoint, the experiments showed that the drift of hysteresis loop being slow, the rate of $\bar{\varepsilon}_{v}$ remained low compared to the rate of the overall strain $\bar{\varepsilon}$. Averaged over a loading cycle, we then estimated that $\tilde{\bar{\varepsilon}}_{e q}$ was representative of the oscillatory strain response.

- Conversely, the rate associated with the equivalent strain, averaged over a complete cycle, named "ratcheting strain rate" $\dot{\bar{\varepsilon}}_{e q}$, was assessed as:

$$
\left(\dot{\tilde{\varepsilon}}_{e q}\right)^{p}=\left(\Delta \tilde{\bar{\varepsilon}}_{e q} \cdot \Delta \tau^{-1}\right)^{p}
$$

where $\left(\Delta \tilde{\bar{\varepsilon}}_{e q}\right)^{p}=\left(\tilde{\bar{\varepsilon}}_{e q}\right)^{p}-\left(\tilde{\bar{\varepsilon}}_{e q}\right)^{p-1}$ and $\Delta \tau^{p}=\tau^{p}-\tau^{p-1}$ represent the increment of the 
equivalent strain per cycle and the time step, respectively. This ratcheting strain rate was considered as representative of the creep strain rate.

\section{Cyclic strain rate:}

Fig. 8 shows 6 fields of the cyclic strain rate captured at different selected fatigue cycles. The spatial distributions were quite heterogeneous in some selected patterns. At the $250^{\text {th }}$ cycle, we observed that the spatial heterogeneities covered the entire gage part, accompanied by low gradients at the upper and bottom ends of the center part. These low gradients continued throughout the loading. SDVs and spatial averages associated with these spatial distributions are pooled in Table 2. According to the statistical findings, the SDVs were clearly all gathered close to the spatial average, with $3 \%$ spatial variations. The average width-wise profiles are also plotted in Fig. 9. It shows that the mean cyclic strain rate values decreased gradually throughout the loading. This decrease was more pronounced at the very beginning of the loading and became slight after reaching the $1000^{\text {th }}$ cycle.

Table 2: Standard deviations and spatial averages associated with the cyclic strain rate maps in Fig. 8.

\begin{tabular}{|l|c|c|c|c|c|c|c|c|}
\hline & $\begin{array}{c}50 \\
\text { cycles }\end{array}$ & $\begin{array}{c}100 \\
\text { cycles }\end{array}$ & $\begin{array}{c}250 \\
\text { cycles }\end{array}$ & $\begin{array}{c}500 \\
\text { cycles }\end{array}$ & $\begin{array}{c}1000 \\
\text { cycles }\end{array}$ & $\begin{array}{c}3000 \\
\text { cycles }\end{array}$ & $\begin{array}{c}5000 \\
\text { cycles }\end{array}$ & $\begin{array}{c}9000 \\
\text { cycles }\end{array}$ \\
\hline$S D \times 10^{-3}\left(\mathrm{~s}^{-1}\right)$ & 3.07 & 3.02 & 2.95 & 2.22 & 2.17 & 2.12 & 2.01 & 1.97 \\
\hline Average $\times 10^{-2}\left(\mathrm{~s}^{-1}\right)$ & 9.64 & 9.51 & 9.16 & 8.49 & 8.15 & 7.86 & 7.60 & 7.38 \\
\hline
\end{tabular}
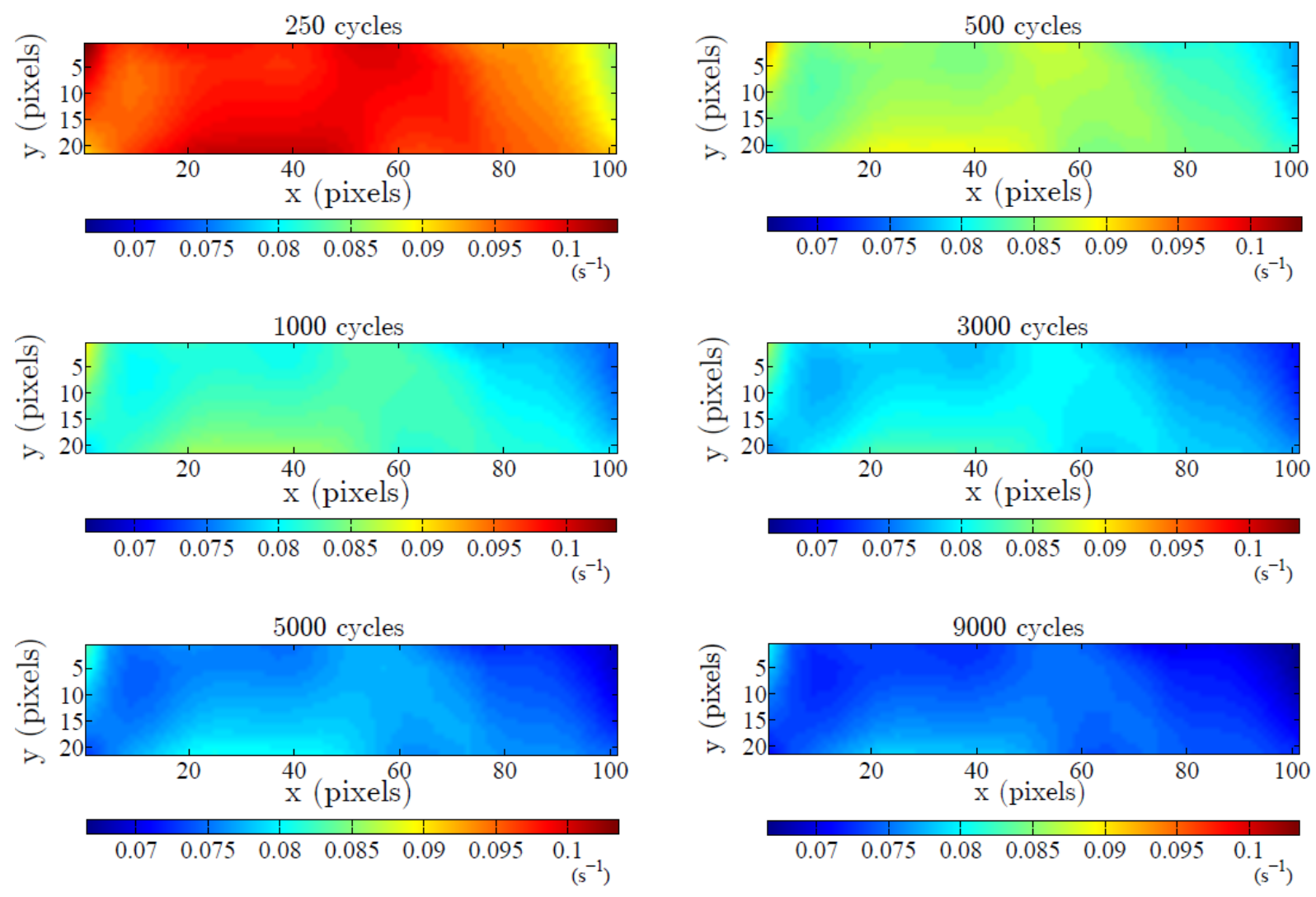

Fig. 8: Maps of the cyclic strain rate computed at different loading stages.

It is interesting to note that even though a slight local maximum appeared close to pixel 50 (where it was previously shown that peak of strain occurred), the cyclic strain rate profile kept its shape throughout the test, slowing down and quasi-stabilizing. 


\section{Ratcheting strain rate:}

Fig. 10 shows the 6 corresponding fields of the ratcheting strain rate. Spatial heterogeneities were clearly visible at the very beginning of the loading. The images performed at the $250^{\text {th }}$ and $500^{\text {th }}$ cycles showed a strong strain rate localization zone at the upper end of the gage length center. From the $1000^{\text {th }}$ cycle, the strain rate localizations started to spatially spread, with the strain rate intensity decreasing.

The statistics associated with these different strain rate distributions are summarized in Table 3. The SDV/spatial average ratio increased at the very beginning of the loading until peaking at $500^{\text {th }}$ cycle, after which it decreased gradually with an increasing number of cycles. The average width-wise profiles shown in Fig. 11 indicated a steep decrease at the very beginning of the loading up to the $500^{\text {th }}$ cycle, and then quasi-stabilized values of around $1.6 \mathrm{~s}^{-1}$. The findings were in close agreement with the observations previously derived from the kinematic analysis of the Hencky strain fields.

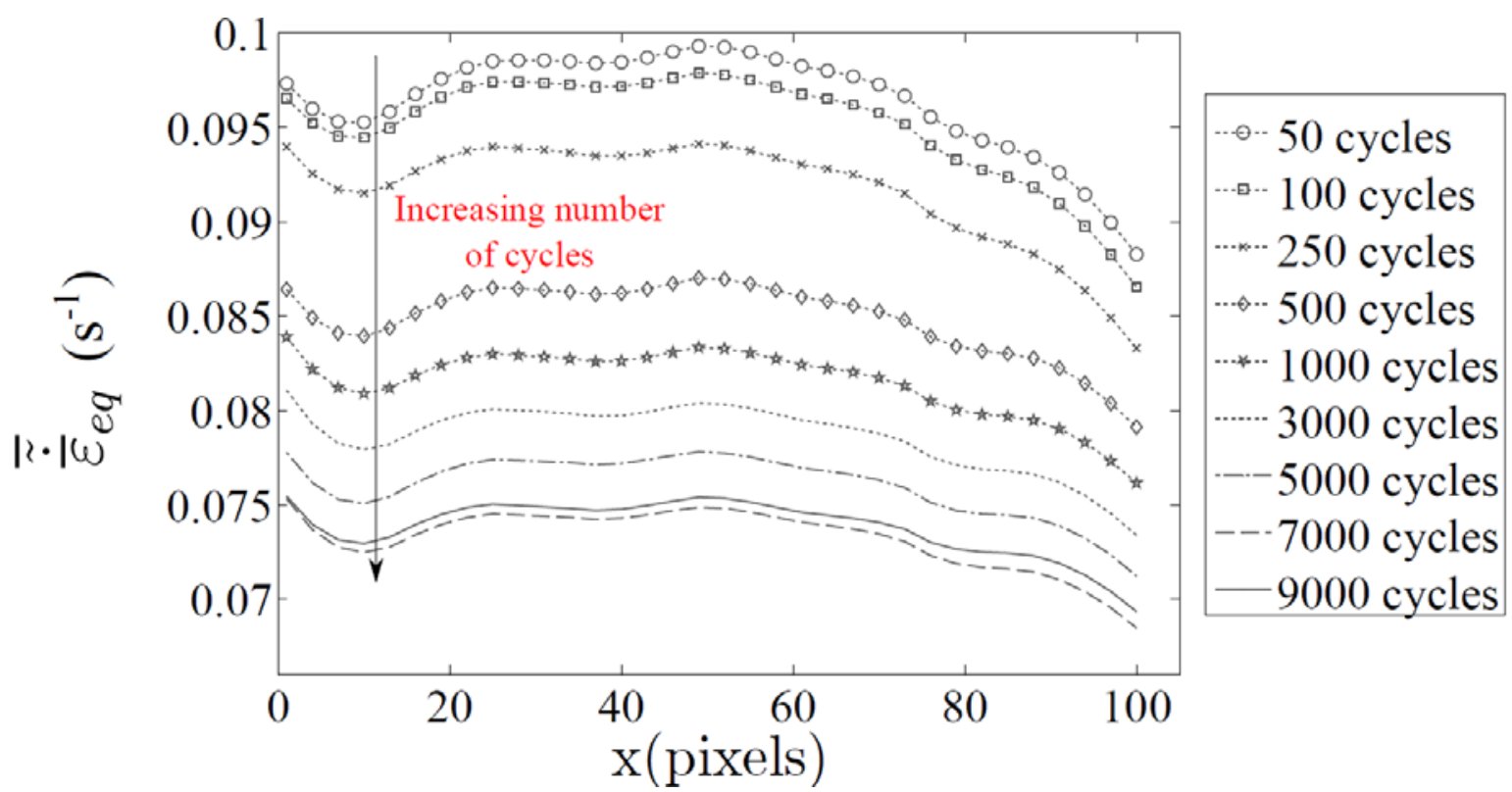

Fig. 9: Average width-wise profiles of the cyclic strain rate as a function of the $x$ coordinate. 

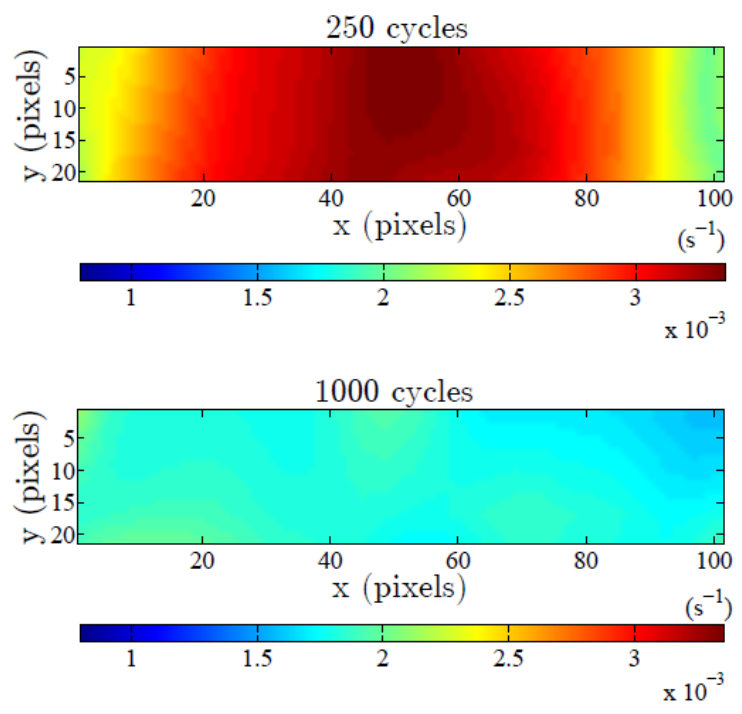

5000 cycles

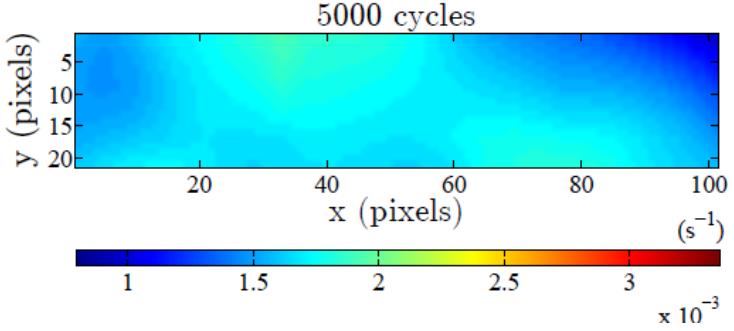

500 cycles
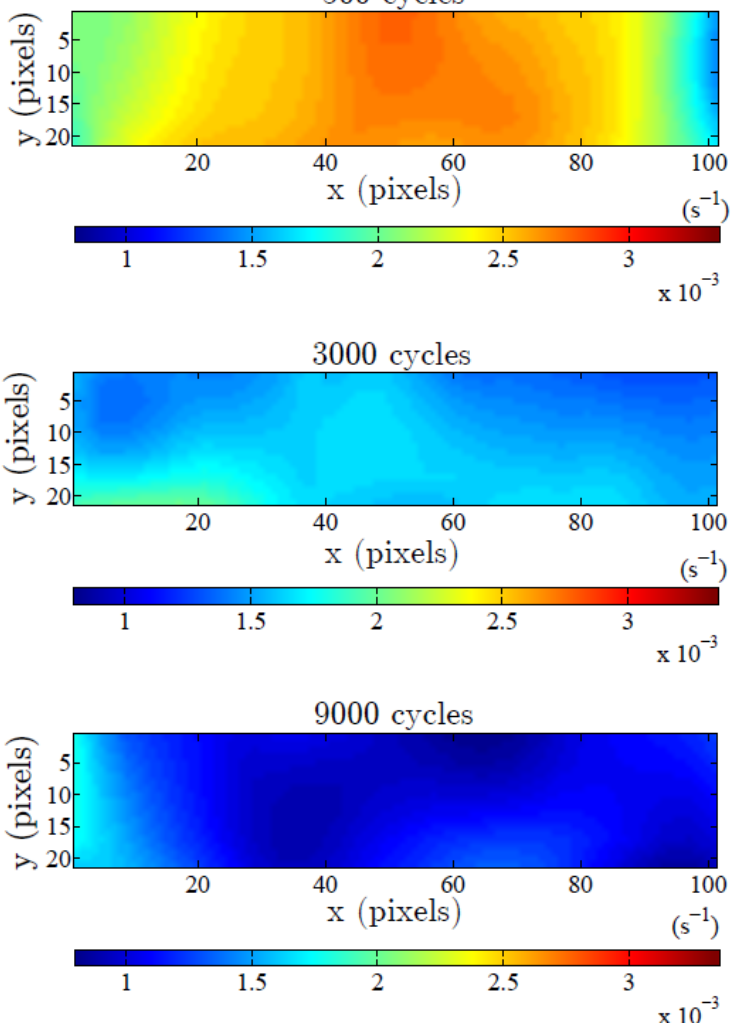

Fig. 10: Maps of the ratcheting strain rate taken at different fatigue stages.

Table 3: Standard deviations and spatial averages associated with the ratcheting strain rate maps shown in Fig. 10.

\begin{tabular}{|l|c|c|c|c|c|c|c|c|}
\hline & $\begin{array}{c}50 \\
\text { cycles }\end{array}$ & $\begin{array}{c}100 \\
\text { cycles }\end{array}$ & $\begin{array}{c}250 \\
\text { cycles }\end{array}$ & $\begin{array}{c}500 \\
\text { cycles }\end{array}$ & $\begin{array}{c}1000 \\
\text { cycles }\end{array}$ & $\begin{array}{c}3000 \\
\text { cycles }\end{array}$ & $\begin{array}{c}5000 \\
\text { cycles }\end{array}$ & $\begin{array}{c}9000 \\
\text { cycles }\end{array}$ \\
\hline$S D \times 10^{-5}\left(\mathrm{~s}^{-1}\right)$ & 10.59 & 27.48 & 38.34 & 32.24 & 6.34 & 6.51 & 6.97 & 7.01 \\
\hline Average $\times 10^{-3}\left(\mathrm{~s}^{-1}\right)$ & 3.88 & 3.43 & 2.94 & 2.48 & 1.85 & 1.64 & 1.62 & 1.11 \\
\hline
\end{tabular}

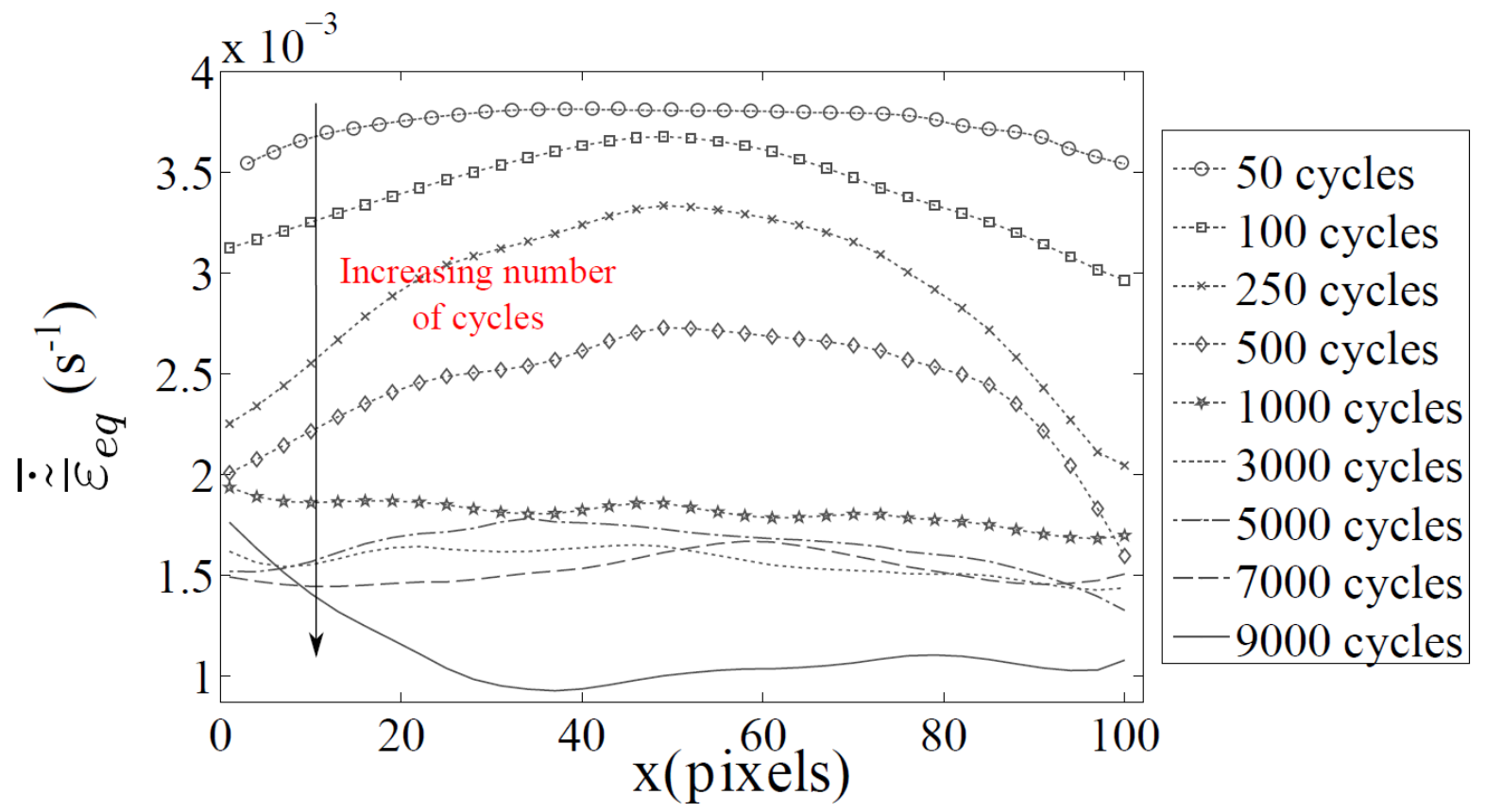


Fig. 11: Average width-wise profiles of the ratcheting strain rate as a function of the $x$ coordinate.

\section{Comparative analysis of kinematical data:}

The analysis of the features of both strain rate distributions highlighted the two following points:

- On the one hand, the difference in order of magnitude of the ratcheting and cyclic strain rates should be underlined. The first one was about $8.10^{-2} \mathrm{~s}^{-1}$, while the second was about $210^{-3}$. The ratcheting strain rate was then approximately 20 to 40 times lower than the cyclic strain rate. These findings matched with the interpretations given for the cyclic and ratcheting strain rates in Eq. (10-11).

- On the other hand, clear localization effects were observed in the ratcheting strain rate patterns, particularly between the beginning of the test and the $1000^{\text {th }}$ cycle, while the distribution of the cyclic strain rate remained rather uniform.

For the sake of simplicity, we can roughly say that the distribution of the cyclic strain rate amplitudes were high and quite uniform while the distribution of ratcheting strain rate amplitudes were low and presented strain concentration effects, particularly before the thousandth cycle. These features will be applied for comparison when analyzing the dissipation patterns.

The next but not least feature to point out was the importance of the ductility of PA6.6 specimens, leading to marked creep effects. We noticed that during preloading of the sample (stage 1) the specimens deformed up to around 7\%, with the highest strains being concentrated in a large central zone of the gage part (cf. Fig. 5-(b)). We then observed that the ratcheting strain developed preferentially in this zone, gradually narrowing up to the thousandth cycle. This phase corresponded to the establishment of the longitudinal strain concentration shown in Fig. 6. Finally, we underlined the slowing down and the progressive standardization of the ratcheting strain rate profile, indicating a quasi-stabilized behavior in the specimen responses (shown in Fig. 7), despite the presence of a clear strain concentration zone. We associated this "regularization effect" with the nature of the microstructure of PA6.6, which is made of numerous interlaced macromolecular chains that probably promotes this non-local behavior.

The dissipation fields were then analyzed in order to estimate the relative importance of the ratcheting and cyclic deformation mechanisms in terms of material degradation. Moreover, although the hygrothermal conditions of PA6.6 should impart a glass transition temperature below the room temperature, the coupling heat source ranges were nevertheless computed to assess the possible coexistence of both standard and entropic thermoelastic coupling effects. The coexistence of "glassy" and "rubber" thermo-signals could naturally be interpreted as material heterogeneity, which could possibly boost the strain concentration development.

\subsection{Heat source analysis}

In this section, we focused on heat source patterns observed during the cyclic tests. Fig. 12 and Fig. 14 depict the spatial distributions of the mean intrinsic dissipation per cycle and the coupling source ranges, respectively. The heat source distributions were estimated using temperature variation maps captured over the corresponding fatigue cycle. Heat sources were 
derived from the integrated form of the heat diffusion equation over the sample thickness (see. Eq. (A12) in Appendix). The reference thermal image was that captured just before loading. The smoothing parameters used for the post-processing of the experimental data were: $N_{x_{T}}=$ $10, N_{y_{T}}=4$ and $N_{t}=10$. The spatial resolution in the heat source patterns was set at $r_{\mathrm{s}}=0.2$ $\mathrm{mm} /$ pixel. Note that the heat sources were divided by the density per unit volume and thus expressed in degrees Celsius per second in order to define an equivalent heating rate to facilitate the comparison between the dissipation and coupling sources. The standard deviations and mean values associated with the selected dissipation and coupling fields are given in Table 4.

Table 4: Standard deviation values and spatial averages associated with the heat source distributions shown in Fig. 12 and Fig. 14.

\begin{tabular}{|c|c|c|c|c|c|c|c|c|c|}
\hline $\begin{array}{c}\text { Heat } \\
\text { sources }\end{array}$ & Statistics & $\begin{array}{c}50 \\
\text { cycles }\end{array}$ & $\begin{array}{c}100 \\
\text { cycles }\end{array}$ & $\begin{array}{c}250 \\
\text { cycles }\end{array}$ & $\begin{array}{c}500 \\
\text { cycles }\end{array}$ & $\begin{array}{l}1000 \\
\text { cycles }\end{array}$ & $\begin{array}{l}3000 \\
\text { cycles }\end{array}$ & $\begin{array}{l}5000 \\
\text { cycles }\end{array}$ & $\begin{array}{c}9000 \\
\text { cycles }\end{array}$ \\
\hline \multirow{2}{*}{$\widetilde{\overline{\mathcal{D}}}_{1} / \rho C$} & $S D \times 10^{-2}\left({ }^{\circ} C \cdot s^{-1}\right)$ & 0.39 & 1.25 & 2.19 & 3.33 & 0.47 & 0.31 & 0.26 & 0.12 \\
\hline & Average $\left({ }^{\circ} \mathrm{C} . \mathrm{s}^{-1}\right)$ & 0.13 & 0.14 & 0.15 & 0.16 & 0.13 & 0.11 & 0.10 & 0.10 \\
\hline \multirow[t]{2}{*}{$\Delta \bar{S}_{c} / \rho C$} & & 0.55 & 0.45 & 0.29 & 0.18 & 0.27 & 0.41 & 0.52 & 0.93 \\
\hline & Average $\left({ }^{\circ} \mathrm{C} . \mathrm{s}^{-1}\right)$ & 2.02 & 1.88 & 1.44 & 1.14 & 1.18 & 1.62 & 1.65 & 2.01 \\
\hline
\end{tabular}

\subsubsection{Maps and profiles of the mean intrinsic dissipation per cycle}

In Fig. 12, a rapid overview of the different fields shows that concentration zones rapidly occurred, increasing in intensity to reach a maximum at the $500^{\text {th }}$ cycle. Then the dissipation intensity decreased drastically and tended towards quasi-homogeneous patterns at the end of the cyclic loading. These concentration zones crossed gradually over the width of the sample gage part. At the $500^{\text {th }}$ cycle, the standard deviation was around $3.33 \times 10^{-2}{ }^{\circ} \mathrm{C}^{-1}{ }^{-1}$ and the mean was approximately equal to $0.16^{\circ} \mathrm{C}^{-1}{ }^{-1}$. This represented around $20 \%$ of the spatial variations. We thus interpreted the rapid development of the highest dissipation zones as a sign of material degradation heralding the strain concentration. Indeed, after the $500^{\text {th }}$ cycle, the intrinsic dissipation values slowly decreased, in accordance with the slowdown in the kinematic data temporal pattern.

The average width-wise dissipation profiles were also plotted in Fig. 13 for the different selected steps. These profiles were used to more precisely locate the highest dissipation value per cross-section and to verify how close it was to the strain concentration maximum. We observed that the maximum dissipation intensity was about $0.2^{\circ} \mathrm{C} . \mathrm{s}^{-1}$ (i.e. $367 \mathrm{~kW} . \mathrm{m}^{-3}$ ) and located at the $56^{\text {th }}$ pixel, while the highest strain value was reached at the $53^{\text {rd }}$ pixel.

At this level, it would be interesting to discuss the time course of the dissipation patterns step by step in association with features of the ratcheting and cyclic strain rate patterns:

- At image \#50, the dissipation distribution was rather uniform even though a slightly higher zone was identified in the $[45,60]$ pixel interval. The same type of distribution is seen in Fig. 11, regarding the ratcheting strain rate profile. Given this low strain rate, the major part of the dissipation was probably due to the deformation energy rate associated with the cyclic strain rate.

- At image \#100, the development of the concentration was now clearly visible. This was also the case for the ratcheting strain rate profiles. Conversely, regarding the cyclic strain rate distributions, the presence of concentration zones is not as clearcut in Fig. 8. 
- From images \#250 to \#500, the intensity of the dissipation in the concentration zones increased while, in parallel, the ratcheting strain rate profiles started to decrease. Associated with this time period, a uniform decreasing shift in the cyclic strain rate profiles was also observed. This unexpected feature led us to interpret the dissipation increase in the concentration zone as being induced by a microstructural degradation without any particular kinematic effects. Note that the dissipation assessments were made based on the assumption that the mass density and the specific heat were material constants.

- From images \#1000 to \#9000, the localized dissipative effects gradually vanished. The dissipation fields tended towards uniform distributions, with the dissipation intensity decreasing regularly. The same types of feature were observed for both the cyclic and ratcheting strain rate.
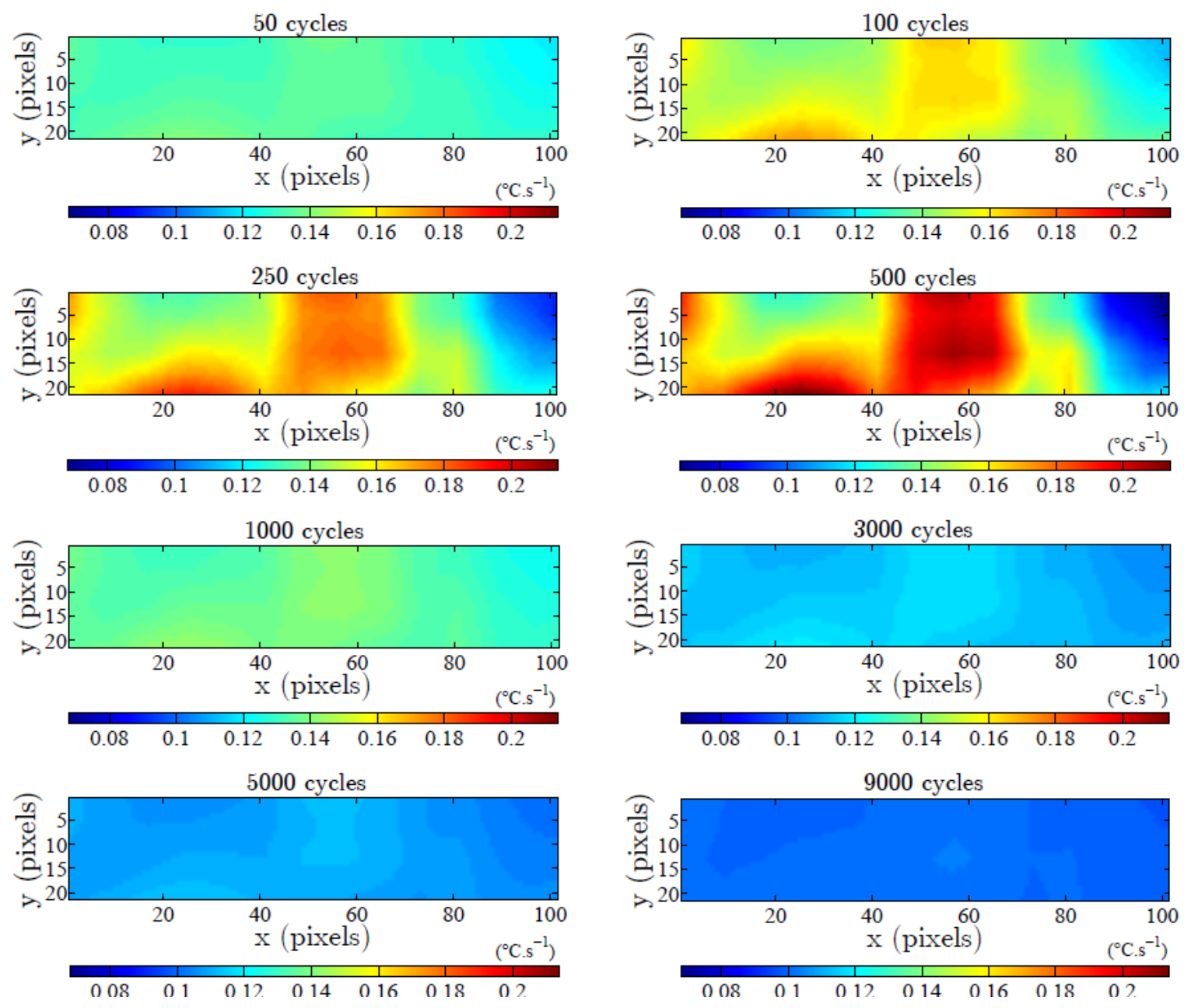

Fig. 12: Maps of the mean intrinsic dissipation per cycle taken at the selected fatigue stages. 


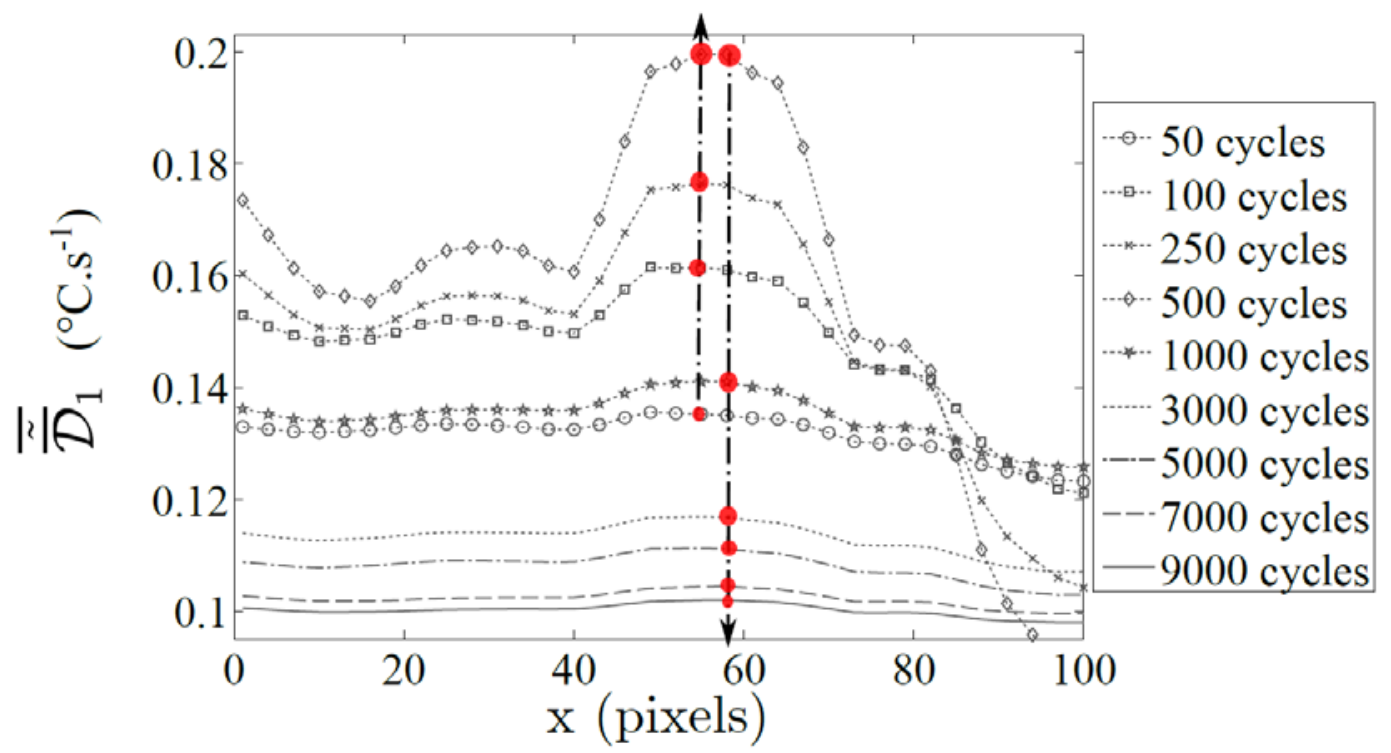

Fig. 13: Average width-wise profiles of the mean intrinsic dissipation per cycle as a function of the x coordinate.

The strong correlation between the development of the ratcheting strain rate and dissipation concentration zones led us to consider that the concentration was mainly induced by heterogeneous development of the so-called creep strains during this asymmetric cyclic loadcontrolled test (i.e. existence of a non-zero mean stress).

At the $500^{\text {th }}$ cycle, we computed an order of magnitude of the deformation energy rate associated with the ratcheting strain rate: it was roughly defined by $\sigma_{\text {mean }} \dot{\overline{\tilde{\varepsilon}}}$ eq where $\sigma_{\text {mean }}$ was the mean stress. Divided by $\rho C$, for a mean stress of about $22 \mathrm{MPa}$ and for a ratcheting strain rate approximately equal to $2.610^{-3} \mathrm{~s}^{-1}$ (cf. Fig.11), we found an energy rate of about $0.03^{\circ} \mathrm{C} . \mathrm{s}^{-}$ 1 . This value was compared with the "peak of over-dissipation" and found of same order of magnitude (cf. Fig.13). This brief analysis, though coarse, was well in the sense of a temporary concentration of dissipative creep strain rates.

Up to the $500^{\text {th }}$ cycle, the initially high strain concentration development then decreased and vanished, here around the thousandth cycle.

From cycles 1000 to 9000 , the cyclic behavior of the specimen gage part became increasingly regular in terms of strain rate and dissipation fields, and this despite the definitive existence of a zone preferentially degraded by concentration mechanisms.

At the $5000^{\text {th }}$ cycle for example, the dissipation field (Fig.12) and profile (Fig.13) were approximately uniform. The dissipation was about $0.11^{\circ} \mathrm{C}^{-1}{ }^{-1}$ and corresponded to the mean intrinsic dissipation averaged over the sample gage part (see Fig.2). So, to go further in the analysis of the behavioral effects, we then reconsidered in Table 5 the energy rate balance at 1 and $10 \mathrm{~Hz}$ averaged over the specimen gage part (Benaarbia et al., 2014a,b), at the end of the cyclic test $\left(10000^{\text {th }}\right.$ cycle) in the same loading conditions and assuming now, a quasi-uniform distribution of a mean energy rate balance per cycle.

Table 5: Comparison of mean energy rate balances per cycle at 1 and $10 \mathrm{~Hz}$, extracted from the overall analysis at the end of the cyclic test $\left(10000^{\text {th }}\right.$ cycle).

\begin{tabular}{|c|c|c|c|}
\hline$f_{L}(\mathrm{~Hz})$ & $\bar{\varepsilon}_{x x}(\%)$ & $\widetilde{w}_{d e f}\left({ }^{\circ}{ }^{\circ} . s^{-1}\right)$ & $\widetilde{\mathcal{D}}_{1}\left({ }^{\circ}{\mathrm{C} . s^{-1}}^{-1}\right.$ \\
\hline 1 & 10 & 0.12 & 0.10 \\
\hline 10 & 4.5 & 0.46 & 0.24 \\
\hline
\end{tabular}


The analysis of thermomechanical data led us to the following comments:

- The mechanical behavior was obviously rate dependent, the drift of the hysteresis loop decreasing approximately by a factor of 2 between 1 and $10 \mathrm{~Hz}$. This finding was found consistent with viscous effects inasmuch as at "high" frequency, the material viscosity limited the ratcheting strain rate when compared with the "low" frequency, for a given stress level.

- The mean dissipation per cycle was greater at "high" strain rate. This result was also compatible with viscous effects. Remember that in the framework of linear viscoelasticity, during a stress controlled monochromatic cyclic test, the mean dissipation per cycle is proportional to the square of the loading frequency for a parallel (Kelvin-Voigt) viscoelastic model, while it remains constant with respect to $f_{L}$ for a series (Maxwell) model. In Table 5, the mean dissipation per cycle is approximately twofold greater at $10 \mathrm{~Hz}$ comparatively to $1 \mathrm{~Hz}$. The PA6.6 behavior does probably not belong the previously mentioned classes of linear viscoelastic model. Non-linear viscous ingredients have to be introduced in the modeling as well as non-kinematic state variables, to take the fatigue damage and water plasticizing effects into account.

- Finally, it is worth noting that the ratcheting strain rate and the mean stored energy per cycle did not disappear until the $10000^{\text {th }}$ cycle. Anelastic shakedown would never occur in such conditions, neither from a mechanical nor thermodynamic standpoint.

\subsubsection{Coupling source amplitude fields and profiles}

As already mentioned, the temperature time variations associated with the cyclic loading of wet PA6.6 essentially consisted of low frequency drift superimposed on oscillations whose frequency spectrum was close to that of the loading (here monochromatic loading). When analyzing the thermosignal over an integer number of cycles, we associated the drift with the dissipative effects and the oscillation ranges with the thermomechanical coupling effects. We also highlighted that two coupling mechanisms could occur, i.e. the standard thermoelastic coupling effects induced by the material thermodilatability and the entropic elasticity induced by the macromolecular chain orientation/disorientation. For a rubber material, under adiabatic conditions, these two coupling heat sources have opposite effects since the first one is in opposite phase to the loading while the second is in phase (see Eq.(6)). As already mentioned, this competition leads to the famous thermoelastic inversion, with the entropic elasticity becoming, from a calorimetric standpoint, predominant at finite strain (Anthony, 1942).

Note that in the case of non-adiabatic conditions, heat diffusion generates a phase shift between the thermosignal and the loading signal and this phase shift may be amplified and distorted by the emergence of a heterogeneous heat source field, which in turn amplifies the local curvatures of the thermal field. Note also that other calorimetric coupling effects may occur for crystallizable natural rubber, induced by the fusion-crystallization transformation (Toki et al., 2000; Samaca et al., 2014). However, this first order solid-solid phase transition was observed at a very high Hencky strain (about 400\%).

Now regarding the PA6.6 behavior, we then limited our interpretation of the thermal oscillations to both thermoelastic effects. Indeed, it is recognized that hygrometric conditions have a marked impact on the glass transition temperature $T_{\mathrm{g}}$. If $T_{\mathrm{g}} \approx 65-70^{\circ} \mathrm{C}$ for dry PA6.6, at $\mathrm{RH}=80 \%$, the glass transition temperature decreases to $T_{\mathrm{g}} \approx 0^{\circ} \mathrm{C}$. As the cyclic tests were performed at a room temperature of about $23^{\circ} \mathrm{C}$, the hygrothermal conditions placed the specimens in a rubbery state. 
Fig. 14-(up) shows the distribution of the thermoelastic coupling source ranges captured around cycle \#100. A first overview shows the heterogeneous but regularly distributed character of the heat source ranges. The contour plots allowed us to roughly sort the different regions of approximately constant range. These were denoted $R \mathrm{i}, L \mathrm{i}, i=1, . ., 4$, and $C 1$. Fig. 14-(down) shows the corresponding temperature variations $T_{c r}$. The data were reduced and centered to facilitate the physical interpretation. We then observed that the temperature signal range decreased from $R_{4}$ to $R_{1}$, with the oscillation being in opposite phase to the loading represented by the black curve, and increased from $L_{1}$ to $L_{4}$, with the oscillation now being in phase with the force signal. In region $C_{1}$, the oscillation range remained low and the frequency spectrum of the signal was more complex than in regions $R_{\mathrm{i}}$ or $L_{\mathrm{i}}$. In $C_{1}$, we supposed that both couplings coexisted, while competing equally.

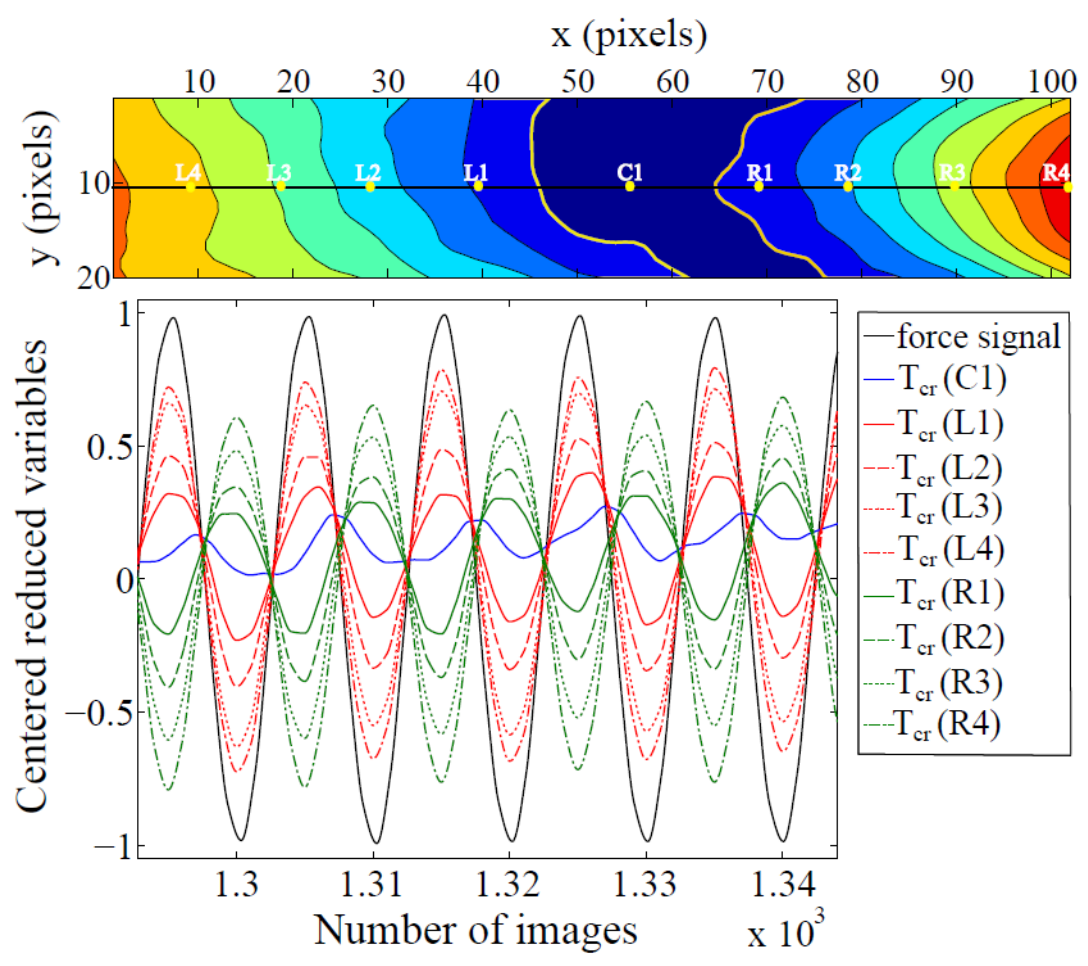

Fig. 14: (up) - Spatial distribution of the thermoelastic coupling source ranges captured around cycle \#100. (down) - Centered reduced signals of the applied force and temperature variations, at regions $R_{\mathrm{i}}, L_{\mathrm{i}}, i=1, . ., 4$, and $C_{1}$, in the form of centered reduced variables highlighting the thermoelastic predominance of regions $R$ and $L$.

In Fig. 15, the coupling source field ranges were plotted at the same steps as those previously chosen for the presentation of strain rates and dissipation fields. At first sight, we can observe an "RCL" partition existing whatever the cyclic number:

- Until cycle \#500, region $C$ increased gradually. The maximum area for region $C$ was reached at cycle \#545, where it covered the whole specimen gage part.

- From here to cycle \#9000, the 3 regions reappeared, with region $R$, associated with the standard thermoelasticity, remaining confined at the extremity of the right hand side of the specimen, i.e. region $L$, associated with entropic elasticity, gradually enlarging.

Note also that the physiognomy of the coupling source distributions differed from those of the strain rates and dissipation. In particular, the strain concentration development, spatially limited by 40 to 70 pixels in length, and temporally limited by cycles \#50 and \#500, was not detectable 
in terms of coupling heat source ranges.

This finding is not very surprising if we consider that the strain concentration was here accompanied by high irreversibility, as shown by the dissipation time patterns, and that the coupling heat sources stemmed from strong interactions between the mechanical, thermal and microstructural states. From a behavioral standpoint, the dissipative effects of the strain concentration have to be described by the evolution equations, while the thermoelastic coupling effects have to be depicted by the state equations. In any case, the co-existence of "glassy", "rubber" and "mixed" regions was an indisputable finding, leading us to consider that PA6.6 was a graded material whose property variations surely influenced the strain concentration development.
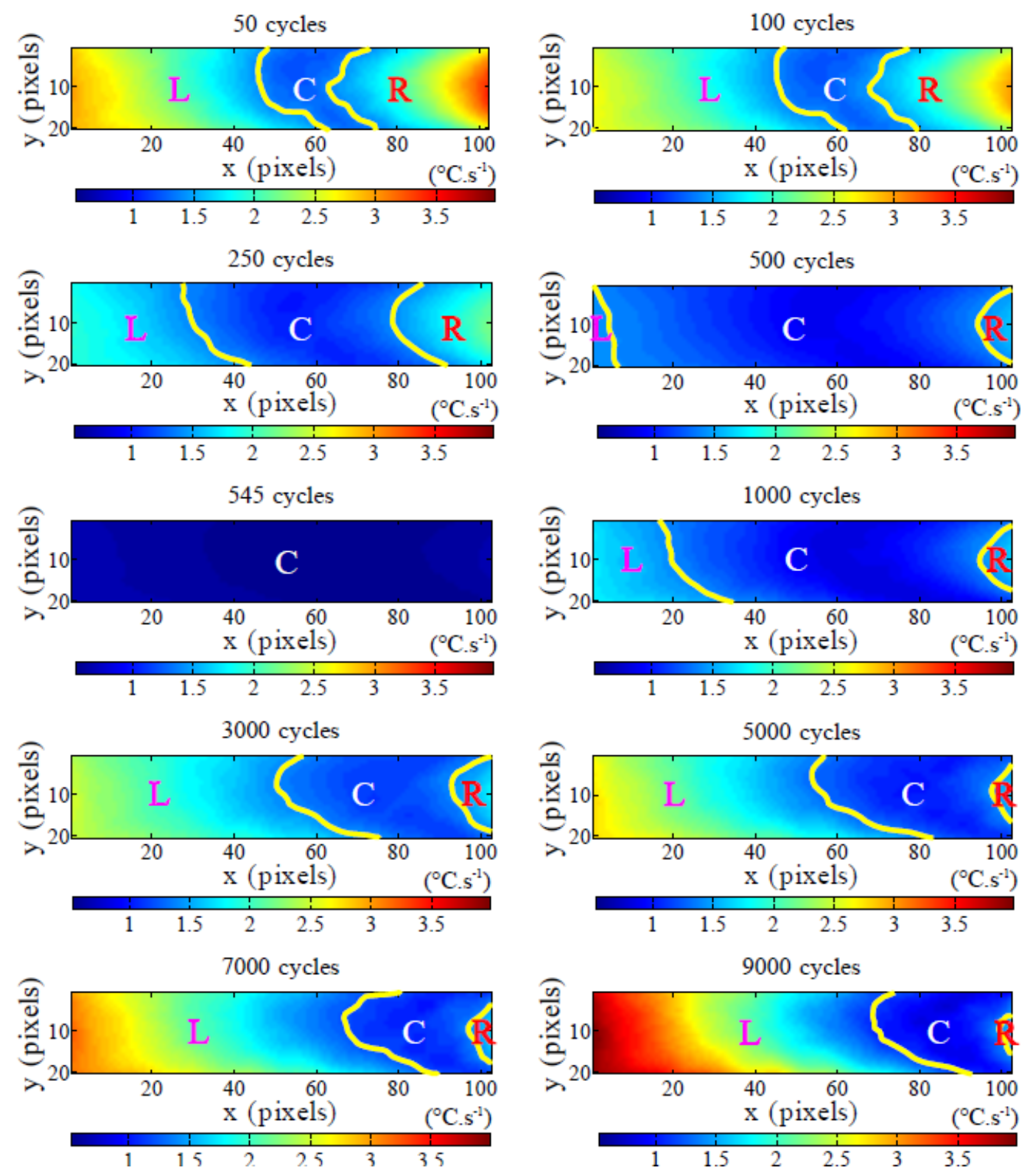

Fig. 15: Maps of the thermomechanical source amplitudes captured at the selected fatigue stages.

\section{Concluding comments}


In this paper, wet PA6.6 specimens were subjected to asymmetric cyclic load-controlled tests at $1 \mathrm{~Hz}$ loading frequency in order to highlight the heterogeneous cyclic patterns of the material thermomechanical responses. Kinematic and calorimetric variables, such as the longitudinal strain, ratcheting and cyclic strain rates, intrinsic dissipation and coupling sources, were systematically computed using photomechanical techniques. Based on an analysis of the test findings, the most noticeable features extracted from the experiments could be summarized as follows:

- Regarding the preloading, we noticed that the specimens deformed up to around 7\%, with slightly marked strain concentration at the center of the gage part. The predeformation intensity highlighted the strong ductility of PA6.6. The locus where the slight pre-concentration appeared became the cyclic strain concentration onset.

- The strain concentration zone progressed in terms of dissipation until the $500^{\text {th }}$ cycle, and then stabilized gradually throughout the rest of the cyclic test.

- In parallel, clear concentration effects were also observed in the ratcheting strain rate patterns, particularly between the beginning of the test and the $500^{\text {th }}$ cycle, while the distribution of the cyclic strain rate remained quite uniform throughout the test. The difference in order of magnitude of the ratcheting and cyclic strain rates was underlined. Although the ratcheting strain rate was approximately 20- to 40 -fold lower than the cyclic strain rate, we associated the "over-dissipation" of the strain concentration zone with the heterogeneous creep effects induced by asymmetric loading.

- The coexistence of glassy and rubber thermo-signals was finally detected and associated with either the material or hygrometric state heterogeneity, which could have promoted the strain concentration development.

- These different results led us to consider that the wet PA6.6 has, at room temperature, a non linear viscoelastic behavior. Parallel and series rheological components have to be introduced to describe the evolution of the stored energy and predict the ratcheting effect. To this modeling ingredients, both standard and entropic thermoelasticity will have to be added. Finally, non-necessarily kinematic state variables describing the precocious development of strain concentration and the role of the water plasticizing effects will propably have to be considered.

We proposed three possible followups to this thermomechanical analysis study:

- The first was associated with the fate of the concentration zone. After a growth phase, the strain concentration zone seemed to stabilize until the end of the test, here limited to $10^{4}$ cycles. It could be interesting to verify if, at the end of the specimen lifetime, the fatigue crack would emanate (or not) from this concentration zone. If so, this would show that the final crack zone could be rapidly determined, thus likely facilitating the formulation of deterministic rules to assess the fatigue lifespan.

- The second was more focused on the industrial concerns. The PA6.6 material used in industrial applications is reinforced with short glass fibers. In previous studies, we already assessed the macroscopical thermomechanical responses of such fiber reinforced PA6.6 composites (Benaarbia et., 2015c). The beginning of the cyclic test 
was also associated with a marked change in the kinematic, thermal and calorimetric parameters used in the present study to characterize the thermomechanical response. It could be of crucial importance to check if the fibers were or not able to restrain the development of such strain concentration during the first thousands of cycles.

- The third dealt with the development of a behavioral model in the framework of the GSM formalism. Currently, a Burgers' assembly was considered as basic structure for the rheological model. This model belongs to the set of linear isothermal viscoelastic models. From a kinematic standpoint, it is in agreement with the strain partition proposed in Eq. (9) and we stress in addition that, once identified, it can give satisfactory predictions of the overall stress-strain responses of the specimen when considering the first dozens of cycles at the beginning of the fatigue test. However the dissipation levels appeared to be systematically too important when compared with the experimental assessments. As previously mentioned this confirmed that the dissipative effects could not be correctly described by linear rheological dash-pot components and highlighted once more that a mechanical model giving satisfactory mechanical predictions, did not give systematically correct thermal and energy predictions. The model has to be enriched by using non linear viscosity components accompanied probably by specialized fatigue state variables able to correctly simulate the heterogeneous energy dissipation and storage accompanying the strain concentration observed during the first thousands of cycles.

\section{Acknowledgements}

The authors gratefully acknowledge Solvay Engineering Plastics for supporting this work and for providing material data and specimens. This work benefited from the financial support of the French Minister for Research (ANRT), CNRS 079212: UM2 121532 and was performed in the framework of the FUI DURAFIP project.

\section{Appendix}

\section{Thermomechanical full-field assessment}

DIC and IRT are optical techniques which have been extensively used in solid mechanics over the last decades (Grediac and Hild, 2012). In some cases, these full-field measurement techniques have enabled researchers to reveal the progressive development of strain concentration zones in which anelastic strains often accumulate, leading to the onset and progress of damage.

\section{A.1. Kinematic estimates}

Full-field measurements of displacements through digital image correlation are very useful for enhancing insight into the physical mechanisms involved during tests conducted on polymeric materials. The DIC technique allows computation of in-plane displacements and displacement gradients by comparing images captured before and after a loading increment. 
However, it is often hard to isolate the strain concentration areas after a direct strain field computation from the raw visible images because of the derivation operations that substantially amplify the noise effects. Signal-processing methods thus had to be developed to reduce noise effects and enhance the kinematic variable estimates. In the following, local least-squares fitting methods were used to estimate the strain and strain rates involved during monochromatic cyclic loadings. In such cases, the local approximation vector function $\overline{\boldsymbol{u}}^{a p p}$ of the noisy 2D displacement data had the following form:

$\overline{\boldsymbol{u}}^{a p p}\left(x_{K}, y_{K}, t\right)=\boldsymbol{\phi}_{1}\left(x_{K}, y_{K}\right)+\boldsymbol{\phi}_{2}\left(x_{K}, y_{K}\right) t+\boldsymbol{\phi}_{3}\left(x_{K}, y_{K}\right) \cos \left(\omega_{L} t\right)+\boldsymbol{\phi}_{4}\left(x_{K}, y_{K}\right) \sin \left(\omega_{L} t\right)$,

where $\omega_{L}$ stands for the loading pulsation, and $\left\{\boldsymbol{\phi}_{n}\right\}_{n=1, \ldots, 4}$ a set of $2 \mathrm{D}$ vectors whose components involve second order polynomials of $x_{K}$ and $y_{K}$ coordinates defined using the following system:

$\boldsymbol{\Phi}=\Xi \aleph_{K}$,

with $\boldsymbol{\Phi}=\left(\boldsymbol{\phi}_{1}, \boldsymbol{\phi}_{2} / f_{a}^{K}, \boldsymbol{\phi}_{3}, \boldsymbol{\phi}_{4}\right)^{t}, \quad \boldsymbol{\Xi}=\left\{\xi_{i, j}\right\}_{4 \times 6}$ being the matrix that contains the coefficients of the vector-valued functions $\boldsymbol{\phi}_{i}$, and $\aleph_{K}=\left(\hat{x}_{K}^{2}, \hat{y}_{K}^{2}, \hat{x}_{K} \hat{y}_{K}, \hat{x}_{K}, \hat{y}_{K}, 1\right)^{t}$ a set of spatial approximation functions taking the geometrical characteristics of the CCD detector element into account, with $\hat{c}_{K}=c_{K} / \Delta c_{K}$ for $c \in\{x, y\}$. The term $f_{a}^{K}$ denotes the CCD frame rate, with the symbol (. $)^{t}$ standing for the transpose of $($.$) .$

Strain tensors can thus be calculated using the deformation gradient $\boldsymbol{I}+\nabla \overline{\boldsymbol{u}}^{a p p}$, where $\boldsymbol{I}$ stands for the identity tensor and $\nabla$ the gradient symbol. Suitable strain tensors must be considered, such as the Hencky strain tensor $\overline{\boldsymbol{\varepsilon}}$, which is calculated using the deformation gradient as follows:

$\overline{\boldsymbol{\varepsilon}}=\frac{1}{2} \ln \left(\left(\boldsymbol{I}+\nabla \overline{\boldsymbol{u}}^{a p p}\right)^{t}\left(\boldsymbol{I}+\nabla \overline{\boldsymbol{u}}^{a p p}\right)\right)$.

For small perturbations, Hencky strain and strain rate tensors can be reduced to linearized strain and strain rate tensors:

$\overline{\boldsymbol{\varepsilon}}=\frac{1}{2}\left(\nabla \overline{\boldsymbol{u}}^{a p p}+\nabla^{t} \overline{\boldsymbol{u}}^{a p p}\right), \dot{\overline{\boldsymbol{\varepsilon}}}=\frac{1}{2}\left(\nabla \dot{\overline{\boldsymbol{u}}}^{a p p}+\nabla^{t} \dot{\overline{\boldsymbol{u}}}^{a p p}\right)$.

The corresponding approximations of the mean Hencky strain components averaged over a complete cycle and computed over a local fitting zone (see. Fig. A1) containing a complete number of loading cycles, with $N_{x_{K}}^{s c} \times N_{y_{K}}^{s c} \times N_{t}^{s c}$ in-dimensions corresponding to the number of pixels over $x_{K}, y_{K}$ and time $t$ can read:

$$
\begin{aligned}
& \tilde{\bar{\varepsilon}}_{x x}=\frac{1}{\Delta x_{K}}\left(2 \xi_{1,1}^{x} \frac{i_{K}}{N_{x_{K}}^{s c}}+\xi_{1,3}^{x} \frac{j_{K}}{N_{y_{K}}^{s c}}+\xi_{1,4}^{x}\right), \\
& \tilde{\bar{\varepsilon}}_{y y}=\frac{1}{\Delta y_{K}}\left(2 \xi_{1,2}^{y} \frac{j_{K}}{N_{y_{K}}^{s c}}+\xi_{1,3}^{y} \frac{i_{K}}{N_{x_{K}}^{s c}}+\xi_{1,5}^{y}\right),
\end{aligned}
$$

and

$$
\tilde{\bar{\varepsilon}}_{x y}=\frac{1}{\Delta y_{K}}\left(\xi_{1,2}^{x} \frac{j_{K}}{N_{y_{K}}^{s c}}+\frac{\xi_{1,3}^{x}}{2} \frac{i_{K}}{N_{x_{K}}^{s c}}+\frac{\xi_{1,5}^{x}}{2}\right)+\frac{1}{\Delta x_{K}}\left(\xi_{1,1}^{y} \frac{i_{K}}{N_{x_{K}}^{s c}}+\frac{\xi_{1,3}^{y}}{2} \frac{j_{K}}{N_{y_{K}}^{s c}}+\frac{\xi_{1,4}^{y}}{2}\right) .
$$


The terms $\xi_{i, j}^{x}$ and $\xi_{i, j}^{y}$ are the longitudinal and vertical components of the vector $\xi_{i, j}$, while $i_{K}$ and $j_{K}$ are defined as follows:

$i_{K}=\sum_{p=-\left(N_{x_{K}}^{s c}-1\right) / 2}^{\left(N_{x_{K}}^{s c}-1\right) / 2}\left\{\hat{x}_{K}\right\}_{p} ; j_{K}=\sum_{m=-\left(N_{y_{K}}^{s c}-1\right) / 2}^{\left(N_{y_{K}}^{s c}-1\right) / 2}\left\{\hat{y}_{K}\right\}_{p}$

The Hencky strain rate components were also derived using the local fitting coefficients as formulated below:

$\tilde{\overline{\bar{\varepsilon}}}_{x x}=\frac{f_{a}^{K}}{\Delta x_{K}}\left(2 \xi_{2,1}^{x} \frac{i_{K}}{N_{x_{K}}^{s c}}+\xi_{2,3}^{x} \frac{\dot{j}_{K}}{N_{y_{K}}^{s c}}+\xi_{2,4}^{x}\right)$,
$\tilde{\overline{\bar{\varepsilon}}}_{y y}=\frac{f_{a}^{K}}{\Delta y_{K}}\left(2 \xi_{2,2}^{y} \frac{j_{K}}{N_{y_{K}}^{s c}}+\xi_{2,3}^{y} \frac{i_{K}}{N_{x_{K}}^{s c}}+\xi_{2,5}^{y}\right)$,

and

$\tilde{\overline{\bar{\varepsilon}}}_{x y}=\frac{f_{a}^{K}}{\Delta y_{K}}\left(\xi_{2,2}^{x} \frac{j_{K}}{N_{y_{K}}^{s c}}+\frac{\xi_{2,3}^{x}}{2} \frac{i_{K}}{N_{x_{K}}^{s c}}+\frac{\xi_{2,5}^{x}}{2}\right)+\frac{f_{a}^{K}}{\Delta x_{K}}\left(\xi_{2,1}^{y} \frac{i_{K}}{N_{x_{K}}^{s c}}+\frac{\xi_{2,3}^{y}}{2} \frac{j_{K}}{N_{y_{K}}^{s c}}+\frac{\xi_{2,4}^{y}}{2}\right)$.

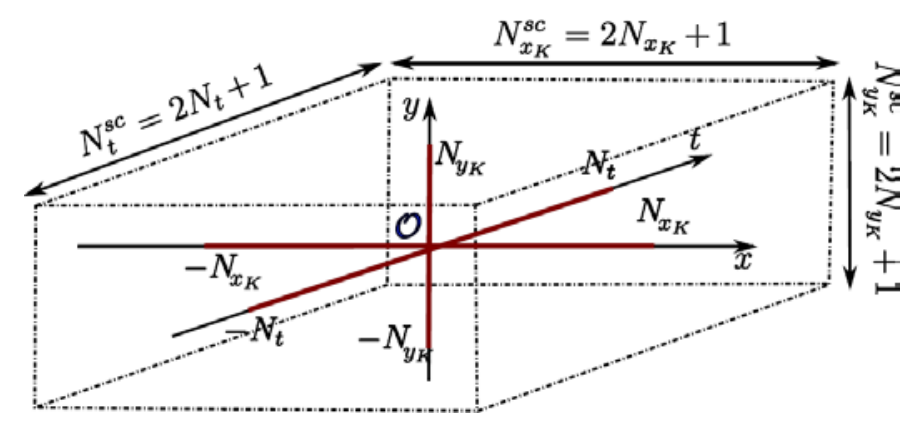

Fig. A1: Geometrical fitting parameters of the local approximation zone.

\section{A.2. Heat source estimate}

IRT is also an optical measurement technique, which has been successfully used for the detection of concentration zones involved during the deformation of polymeric materials (see e.g. Wattrisse et al., 2002; Moreau et al., 2005). This technique is based on the measurement of IR radiation emitted by the loaded samples, with the link between IR radiation and the temperature being determined according to Planck's law.

When a material undergoes mechanical loading, it dissipates part of the deformation energy. This dissipated part generates a progressive increase in material temperature changes. These changes are not intrinsically related to the material behavior because they are affected by heat transfers. It is therefore essential to find alternative ways for evaluating more intrinsic data representative of the material behavior, such as heat sources. These latter can be estimated on the basis of partial derivative operators present in the local form of the heat diffusion equation. For thin flat specimens and by averaging the $3 \mathrm{~d}$ heat equation over the sample thickness, a $2 \mathrm{~d}$ thermal diffusion model can be obtained. It reads:

$\rho C\left(\frac{\partial \bar{\theta}}{\partial t}+\boldsymbol{v} \cdot \nabla \bar{\theta}+\frac{\bar{\theta}}{\tau_{t h}^{2 D}}\right)-k \Delta_{2} \bar{\theta}=\overline{\mathcal{D}}_{1}+\bar{s}_{C}$, 
where $\rho, k$ and $C$ represent the material density, conduction coefficient and specific heat, respectively. The term $\Delta_{2}$ represents the 2D Laplacian operator, while $\bar{\theta}, \overline{\mathcal{D}}_{1}$ and $\bar{s}_{c}$ in turn stand for the temperature variations, intrinsic dissipation and coupling sources averaged over the sample thickness. The parameter $\tau_{t h}^{2 D}$ represents the time constant characterizing perpendicular heat exchange between the outer in-plane surfaces of the specimen and the surroundings. Note that the convective terms corresponding to the scalar product of the displacement velocity $\boldsymbol{v}$ and temperature gradients $\boldsymbol{\nabla} \bar{\theta}$ in the total time derivative of the temperature are often assumed to be negligible. This hypothesis is naturally questionable for heterogeneous polymeric materials where strong spatial concentrations can occur. In previous studies, it was shown that the contribution of $\rho C \boldsymbol{v} \cdot \boldsymbol{\nabla} \bar{\theta}$ could represent around 30\% of the overall heat sources when computed in the propagating necking lips of some polymeric materials (Wattrisse et al., 2002).

When using the same image processing technique as that used for the kinematic data estimates, the local approximation function $\theta^{a p p}$ of the thermal data had the following form:

$\bar{\theta}^{\text {app }}\left(x_{T}, y_{T}, t\right)=\psi_{1}\left(x_{T}, y_{T}\right)+\psi_{2}\left(x_{T}, y_{T}\right) t+\psi_{3}\left(x_{T}, y_{T}\right) \cos \left(\omega_{L} t\right)+\psi_{4}\left(x_{T}, y_{T}\right) \sin \left(\omega_{L} t\right)$,

where $\left\{\psi_{n}\right\}_{n=1, \ldots, 4}$ represents a set of second order polynomials of $x_{T}$ and $y_{T}$ coordinates defined using the following system:

$\boldsymbol{\Psi}=\mathbf{K} \aleph_{T}$,

with $\boldsymbol{\Psi}=\left(\psi_{1}, \psi_{2} / f_{a}^{T}, \psi_{3}, \psi_{4}\right)^{t}, \mathbf{K}=\left\{\kappa_{i, j}\right\}_{4 \times 6}$ being the matrix that contains coefficients of the functions $\psi_{i}$, and $\aleph_{T}=\left(\hat{x}_{T}^{2}, \hat{y}_{T}^{2}, \hat{x}_{T} \hat{y}_{T}, \hat{x}_{T}, \hat{y}_{T}, 1\right)^{t}$ being a set of spatial approximation functions taking the geometrical characteristics of the IR detector element into account. The term $f_{a}^{T}$ stands for the IR frame rate.

In Eq. (14), the trigonometric time functions describe the periodic part of the coupling effects while the linear part is associated with the local approximation (finite development of order one) of the transient effects induced by dissipation heating and possible equilibrium temperature drifts. It is worth underlining that the frequency spectrum of the coupling sources was supposed to be the same as that of the loading. The corresponding approximations of the heat sources (e.g. mean intrinsic dissipation per cycle and coupling source ranges) over a local fitting zone containing a complete number of loading cycles, with $N_{x_{T}}^{s c} \times N_{y_{T}}^{s c} \times N_{t}^{s c}$ in-dimensions corresponding to the number of pixels over $x_{T}, y_{T}$ and time $t$, can read (Boulanger, 2004):

$\frac{\widetilde{\mathcal{D}}_{1}}{\rho C}=\left(\frac{\kappa_{2,1}}{\Delta t_{T}}+\frac{\kappa_{1,1}}{\tau_{t h}^{2 D}}\right) \frac{i_{T}^{2}}{N_{x_{T}}^{S C}}+\left(\frac{\kappa_{2,2}}{\Delta t_{T}}+\frac{\kappa_{1,2}}{\tau_{t h}^{2 D}}\right) \frac{j_{T}^{2}}{N_{y_{T}}^{S C}}+\left(\frac{\kappa_{2,6}}{\Delta t_{T}}+\frac{\kappa_{1,6}}{\tau_{t h}^{2 D}}\right)-\frac{2 k}{\rho C}\left(\frac{\kappa_{1,1}}{\Delta x_{T}^{2}}+\frac{\kappa_{1,2}}{\Delta y_{T}^{2}}\right)$,

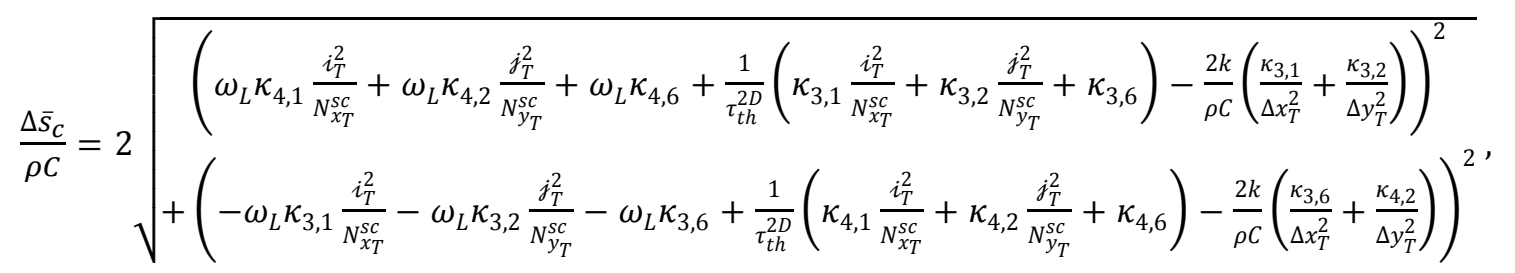

where $\left(\Delta t_{T}\right)^{-1}=f_{a}^{T}$. The terms $i_{T}$ and $j_{T}$ are defined as follows: 
$i_{K}=\sum_{p=-\left(N_{x_{T}}^{s c}-1\right) / 2}^{\left(N_{x_{T}}^{s c}-1\right) / 2}\left\{\hat{x}_{T}\right\}_{p} ; j_{K}=\sum_{m=-\left(N_{y_{T}}^{s c}-1\right) / 2}^{\left(N_{y_{T}}^{s c}-1\right) / 2}\left\{\hat{y}_{T}\right\}_{p}$

These formulations were deduced from the 2D linear heat diffusion equation. Readers interested in the image processing aspects of these infrared thermography techniques associated with the computation of heat sources are referred to Berthel (2007), Benaarbia (2014d).

\section{References}

Benaarbia, A., Chrysochoos, A., Robert, G., 2014b. Influence of relative humidity and loading frequency on the PA6.6 cyclic thermomechanical behavior: Part I. Mechanical and thermal aspects. Polymer Testing. 40, 290-298.

Benaarbia, A., Chrysochoos, A., Robert, G., 2014a. Kinetics of stored and dissipated energies associated with cyclic loadings of dry polyamide 6.6 specimens. Polymer Testing. 34, 155-167.

Arif, M.-F., Meraghni, F., Chemisky, Y., Despringre, N., Robert, G., 2014. In situ damage mechanisms investigation of PA66/GF30 composite: Effect of relative humidity. Composites Part B: Engineering. 58, 487-495.

Horst, J.-J., Spoormaker, J.-L., 1996. Mechanisms of fatigue in short glass fiber reinforced polyamide 6. Polymer Engineering \& Science. 36, 2718-2726.

Bernasconi, A., Kulin, R.-M., 2009. Effect of frequency upon fatigue strength of a short glass fiber reinforced polyamide 6: A superposition method based on cyclic creep parameters. Polymer Composites. 30, 154-161.

Launay, A., Maitournam, M.-H., Marco, Y., Raoult, I., Szmytka, F., 2011. Cyclic behaviour of short glass fibre reinforced polyamide: Experimental study and constitutive equations. International Journal of Plasticity. 27, 1267-1293.

Launay, A., Marco, y., Maitournam, M.-H., Raoult, I., 2013. Modelling the influence of temperature and relative humidity on the time-dependent mechanical behavior of a short glass fibre reinforced polyamide. Mechanics of Materials. 56, 1-10.

Grediac, M., Hild., F., 2012. Full-Field Measurements and Identification in Solid Mechanics. Wiley.

Bever, M.-B., Holt, D.-L., Titchener, A.-L., 1972. The stored energy of cold work. Progress in Materials Science. 17, 1-192.

Chrysochoos, A., Dulieu-Barton, J., 2015. Infra-red techniques for thermomechanical characterisation of materials and structures. Experimental mechanics. 55, 651-652.

Chrysochoos, A., Maisonneuve, O., Martin, G., Caumon, H., Chezeaux, J.-C., 1989. Plastic and dissipated work and stored energy. Nuclear Engineering and Design. 114, 323-333.

Chrysochoos, A., Louche, H., 2000. An infrared image processing to analyse the calorific effects accompanying strain localization. International Journal of Engineering Science. 38, 1759-1788.

Brandrup, J., Immergut, E.-H., Grulke, E.-A., 1999. Polymer handbook. $4^{\text {th }}$ ed., J. Wiley \& Sons., Inc., New York.

Sperling, L.-H., 1992. Introduction to Physical Polymer Science. 2nd ed., J. Wiley \& Sons., Inc., New York.

Rodriguez, F., 1996. Principles of Polymer Systems. 4th ed. Taylor \& Francis, Washington, DC, Chapter 13 (Recycling and Resource Recovery). 
Joule, W., 1857. On some thermodynamic properties of solids. Philosophical Magazine, Taylor \& Francis, 4th Ser., 14, 227.

Treloar, L.-R.-G., 1973. The elasticity and related properties of rubbers. Reports on Progress in Physics. 36, 755-826.

Treloar, L.-R.-G., 1975. The physics of rubber elasticity. 3rd edit., Clarendon press, Oxford.

Flory, P.-J., Erman, B., 1982. Theory of elasticity of polymer networks. Macromolecules. 15, 800-806.

Chadwick, P., Creasy, C.-D.-M., 1984. Modified entropic elasticity of rubberlike materials. Journal of Mechanics of Physics and Solids. 32, 337-397.

Arruda, E.-M., Boyce, M.-C., 1993. A three-dimensional model for the large stretch behavior of rubber elastic materials. Journal of Mechanics and Physics of Solids. 41, 389-412.

Germain P., Nguyen, Q., S., Suquet, P., 1983. Continuum thermomechanics. Journal of Applied Mechanics. 50, 1010-1020.

Blanche, A., Chrysochoos, A., Ranc, N., Favier, V., 2014. Dissipation Assessments During Dynamic Very High Cycle Fatigue Tests. Experimental Mechanics. 55, 699-709.

Benaarbia, A., Chrysochoos, A., Robert G., 2014c. Influence of relative humidity and loading frequency on the PA6.6 cyclic thermomechanical behavior: Part II. Energy aspects. Polymer Testing. 41, 92-98.

Halphen, B., Nguyen, Q., S., 1975. On the generalized standards materials (in French). Journal de Mécanique, 14, 39-63.Honorat, V., Moreau, S., Muracciole, J.-M., Wattrisse, B.,

Chrysochoos, $\quad$ A., 2005. Calorimetric analysis of polymer behaviour using a pixel calibration of an IRFPA camera. Qirt Journal. 2, 153-171.

Berthel, B., 2007. Dissipation field measurements accompanying the high cycle fatigue of steel by infrared thermography (in French). University of Montpellier II, Sciences et Techniques du Languedoc, Thesis.

Benaarbia, A., 2014d. Energy analysis of the thermomechanical behavior of pure and short glass fiber-reinforced PA6.6 (in French). University of Montpellier II, Sciences et Techniques du Languedoc, Thesis.

Wattrisse, B., Muracciole, J.-M., Chrysochoos, A., 2002. Thermomechanical effects accompanying the localized necking of semi-crystalline polymers. International Journal of Thermal Sciences. 41, 422-427.

Moreau, S., Chrysochoos, A., Muracciole, J.-M., Wattrisse, B., 2005. Analysis of thermoelastic effects accompanying the deformation of PMMA and PC polymers. Comptes rendus Mécaniques. 333, 648-653.

Boulanger, T., 2004. Infrared thermography analysis of heat sources induced by the fatigue of steels (in French). University of Montpellier II, Sciences et Techniques du Languedoc, Thesis.

Toki, S., Fujimaki, T., Okuyama, M., 2000. Strain induced crystallization of natural rubber as detected real time by wide angle X-ray diffraction technique. Polymer. 41, 54235429.

Martinez, S., Ricardo, J., Balandraud, X., Toussaint, E., 2014. Thermomechanical analysis of the crack tip zone in stretched crystallizable natural rubber by using infrared thermography and digital image correlation. Polymer. 55, 6345:6353.

Benaarbia, A., Chrysochoos, A., Robert G., 2015c. Thermomechanical behavior of PA6.6 composites subjected to low cycle fatigue. Composites Part B. 76, 52-64. 